\title{
Uncertainty and Global Sensitivity Analysis of Neutron Survival and Extinction Probabilities Using Polynomial Chaos
}

\author{
C.M. Cooling ${ }^{1}$, D.A.F. Ayres ${ }^{1}$, A.K. Prinja ${ }^{1,2}$, M.D. Eaton ${ }^{1}$ \\ ${ }^{1}$ Nuclear Engineering Group, Department of Mechanical Engineering, Exhibition Road, South \\ Kensington Campus, Imperial College London, SW7 2AZ, UK \\ ${ }^{2}$ Department of Nuclear Engineering, University of New Mexico, Albuquerque, NM 87131, USA
}

\begin{abstract}
Generalised Polynomial Chaos (GPC) in conjunction with sparse grid stochastic collocation and High Dimensional Model Representation (HDMR) is used to perform uncertainty and global sensitivity analysis for the neutron chain survival and extinction probabilities, with and without an intrinsic random source. Starting with a lumped backward Master equation formulation, uncertainty is introduced by allowing the factorial moments of the fission multiplicity distribution, the neutron lifetime, and strength of the intrinsic source to be independent and uniformly distributed random variables. A multidimensional Legendre chaos representation of the random survival and extinction probabilities is used to achieve optimal numerical convergence in the stochastic dimension and the relative variance contributions from each random parameter are then quantified using HDMR.

The underlying deterministic results of the model are found to closely match analytical benchmarks and, once uncertainty is introduced, the GPC results match both Monte Carlo simulations and analytical results for polynomial order greater than two. The GPC method is found to require significantly less computational time to achieve a given accuracy on the survival and extinction probabilities than the Monte Carlo method. It is found that, the probabilities are most sensitive to $\chi_{i}$ for lower $i$ and have a significant sensitivity to $\chi_{2}$ in all cases. A chain's survival probability is moderately sensitive to the neutron lifetime early in simulation. In the subcritical case this sensitivity increases as the simulation continues whilst it decreases in the supercritical case. The extinction probability is sensitive to the source strength.
\end{abstract}

Keywords: Extinction Probability, Backward Master Equation, Polynomial Chaos, Global Sensitivity

\section{Introduction}

A vast body of work has been published to date on the probabilities of extinction, survival and divergence of the neutron population in a multiplying medium Bell

Email address: c.cooling10@imperial.ac.uk (C.M. Cooling $\left.{ }^{1}\right)$ 
(1963, 1965); Williams (1974); Pazsit and Pal (2008); Prinja and Souto (2009, 2010); Prinja (2012). These probabilities are key quantities in the characterization of strongly stochastic neutronic systems (exemplified by multiplying media with weak internal sources) where the fluctuations in the neutron number are large enough that low order statistical moments, such as the mean and variance of the neutron number, do not provide sufficient information to describe the instantaneous state of the neutron population and to predict its time evolution. Applications where such stochastic behaviour is important include: criticality excursions in spent fuel storage, in the handling of fissile solutions in fuel fabrication and reprocessing; approach to critical under suboptimal reactor start-up conditions; preinitiation in fast burst research reactors; and weak nuclear signatures in the passive detection of nuclear materials. Especially from a safety viewpoint, it is clearly important to have an accurate estimate of the probability that a neutron chain will grow without bound.

The subject of stochastic neutron populations lends itself to an elegant and complete formulation based on the theory of discrete state, continuous time Markov processes. For point or lumped systems, Master equations of the forward and backward type can be derived for the probability of finding a neutron population of a certain size at a certain time Bell (1963, 1965); Williams (1974); Pazsit and Pal (2008); Prinja and Souto (2009); Prinja (2012). Both approaches yield systems of differential-difference equations for the neutron number Probability Density Function (PDF), with one distinction being that the backward Master equation is nonlinear in the PDF while the forward equation is linear. A key advantage of the backward formulation is that it is not necessary to first obtain the complete neutron number distribution from which to then extract the extinction or survival probabilities, as is the case with the forward approach. Starting with the backward Master equation written in terms of the generating function, explicit and closed, albeit nonlinear, differential equations for the extinction and survival probabilities can be directly written down Bell (1965); Pazsit and Pal (2008); Prinja (2012). Moreover, this can be done for both the lumped case and unlumped case where the neutron phase space dependence is also included. The backward formulation therefore allows accurate numerical calculation of these probabilities under quite general conditions while also enabling analytical solutions to be constructed when the physical model can be simplified.

What appears not to have been addressed in the literature is a systematic quantification of the effects of uncertainty in the physical parameters on the extinction and survival probabilities. The principal parameters with respect to which uncertainty analysis would contribute to a better appreciation of the impact of growth and termination of chains are nuclear data - cross sections, fission neutron multiplicities, and the strength of the intrinsic neutron source (e.g, spontaneous fission rate) - and, in a lumped model, gross parameters such as the neutron lifetime as well as the system reactivity. The relatively small number of compilations of multiplicity data for fissile materials in the open literature confirm the large uncertainties in the different measurements reported (Diven et al., 1956; Zucker and Holden, 1986; Orndoffl, 1957). On the other hand, considerable effort has been devoted over the years to reducing cross section data uncertainty for the important fissile materials. However, the intrinsic source strength is generally not known with good accuracy (Radkowsky, 1964) and 
the uncertainty in the neutron lifetime is potentially large as effects of physical geometry, material heterogeneity, and energy dependence are collapsed and encapsulated in a single parameter for use in a one speed point model. Moreover, depending on the application, the system reactivity may also be subject to uncertainty.

In this paper we use a lumped backward model to carry out a detailed numerical investigation of the effect of physical parameter uncertainty on the time-dependent single neutron chain survival probability and the time-dependent extinction probability when an intrinsic random source is present. Specifically, uncertainty is introduced in the factorial moments of the fission neutron multiplicity distribution, the source strength and the neutron lifetime, and all parameters are assumed to be independent random variables each uniformly distributed about their respective reference values. Strictly speaking, the uncertainty should be introduced directly into the fission neutron multiplicities and propagated into the factorial moments but the constraint that the multiplicity distribution be normalized correlates the multiplicities and significantly complicates the uncertainty analysis. Although stochastic UQ methods of the type considered here can be readily extended to accommodate correlated, Gaussian distributed input variables, more advanced techniques are necessary to represent correlated non-Gaussian distributed input variables (Park et al., 2015). For this reason we have, in this preliminary investigation, incorporated uncertainty directly into the factorial moments, source strength and neutron lifetime and defer a more rigorous representation of uncertainty in the multiplicities to a more comprehensive future investigation. The multiplication factor or k-eigenvalue is generally considered to be an accurately known parameter for use in lumped systems, unlike the intrinsic source and neutron lifetime, so it is not treated as an uncertain variable this work. We emphasize that this does not represent a limitation of the methodology as it simply adds an extra independent random variable, albeit at increased computational cost.

We further emphasize that one goal of this investigation is to consider the effect of uncertainty due to the lumping of distributed systems, which constitutes another layer of uncertainty over the uncertainty in underlying cross sections and numerical approximation schemes. This arises because in the derivation of any value for the lumped system an assumption regarding the neutron flux in terms of space, energy and angle must be made in order to perform the appropriate averaging. However, particularly when the neutron population is very low (as is the case in the systems studied in this paper) the neutron flux is inherently stochastic in this regard which introduces a further uncertainty beyond that of the uncertainty of the neutronics data of the medium in which the neutrons exist. A rigorous treatment of uncertainty propagation in the neutron chain probabilities of interest here, one where uncertainty can be introduced in the fundamental measured parameters (cross sections, multiplicities), must be based on an unlumped backward Master equation formulation that accommodates space, energy and angle dependence and, although merited, such a study would be computationally very demanding and beyond the scope of the present investigation. We hope to report on a more thorough investigation along these lines at a later date.

The polynomial chaos method is used to express the output uncertainty in a spectral expansion and stochastic collocation applied to obtain and solve uncoupled equations for the deterministic expansion coefficients. This representation gives a full statisti- 
cal characterization of the uncertainty in the survival and extinction probabilities of interest here from which statistical moments as well as PDFs are extracted in post processing. Finally, a global sensitivity analysis is performed using High Dimensional Model Representation (HDMR) which yields the relative contribution of each random variable to the total variance. We emphasize that these results are not subject to the restriction of linear sensitivity theory and hence provide a reliable quantification of the sensitivity to each input parameter.

The scope of the paper is as follows. In the next section we present the backward model for the survival and extinction probabilities, provide the reference data, and give a closed form analytical solution for a special case that is useful for benchmarking the numerical solution method used. Numerical solutions for baseline or reference parameters are presented and discussed in the following section. A general framework for conducting uncertainty analysis is then presented along with analytical solutions for the PDFs of the unknowns in a special case. The generalized polynomial chaos method for uncertainty quantification is introduced in the next section and detailed numerical results and analysis from the implementation of this method using nonintrusive sparse grid quadrature methods and global sensitivity analysis are presented. We present some concluding remarks in the final section.

\section{Backward Equations for the Extinction/Survival Probability}

The backward equations for the single chain survival probability $P(t)$ and the extinction probability for random source sponsored chains $P_{E}(t)$ are well known Bell (1965); Pazsit and Pal (2008); Prinja (2012) and may be expressed as:

$$
\begin{aligned}
\frac{\partial P(t)}{\partial t} & =\left(\frac{k_{\mathrm{eff}}-1}{\tau}\right) P(t)-\frac{p_{f}}{\tau} \sum_{i=2}^{\chi_{\max , f}}(-1)^{i} \frac{\chi_{i, f}}{i !}[P(t)]^{i}, \quad P(0)=1, \\
\frac{\partial P_{E}(t)}{\partial t} & =S_{0}\left[\sum_{i=1}^{\chi_{\max , s}}(-1)^{i} \frac{\chi_{i, s}}{i !} P^{i}(t)\right] P_{E}(t), \quad P_{E}(0)=1 .
\end{aligned}
$$

In the above, $\tau$ is the neutron lifetime, $p_{f}$ is the probability of fission, $k_{\text {eff }}$ is the system multiplication factor, $S_{0}$ the intrinsic source strength, $\chi_{i, f}$ and $\chi_{i, s}$ are the factorial moments of the neutron multiplicity distribution for fission and the source respectively and $\chi_{\max , f}$ and $\chi_{\max , s}$ define where the relevant sums are truncated. These values are not, in general, required to eb identical but, for the systems we describe in this paper, we assume the source and fission have the same neutron multiplicity distribution (i.e. the source is a fission source) and so $\chi_{i, f}=\chi_{i, s}$ which we will henceforth simply call $\chi_{i}$ and $\chi_{\max , f}=\chi_{\max , s}$ which we will henceforth refer to as $\chi_{\max } \cdot \chi_{i}$ are defined by:

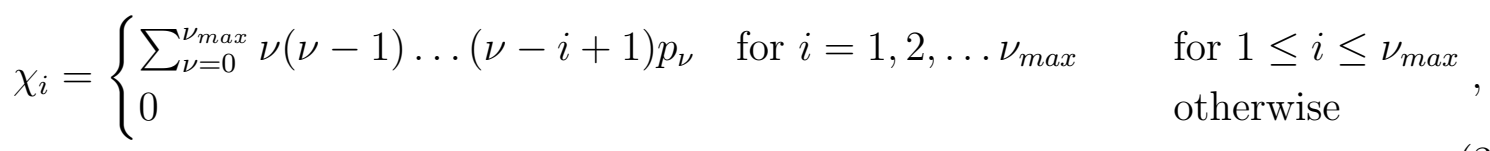

where $p_{\nu}$ is the probability of $\nu$ neutrons being emitted in a fission reaction and $\nu_{\max }$ is the maximum number of neutrons emitted by a fission being considered. It then 
follows that $p_{f}=k_{\text {eff }} / \bar{\nu}$, where $\bar{\nu}=\chi_{1}$ is the mean number of neutrons. Note that this definition sets the limit that $\chi_{\max } \leq \nu_{\max }$. Throughout this article we will set $\nu_{\max }=7$.

The multiplicity term in Equation (1) represents independent chains created by neutrons born in induced fission reactions while the corresponding term in Equation(2) refers to neutrons produced in spontaneous fission. Although in principle the induced and spontaneous fission multiplicities may differ, we have assumed them to be identical.

For the purposes of this investigation, the system parameters are assumed to be static, that is, time independent. This gives the simplification that the resulting survival and extinction probabilities depend only on the difference between final and initial times as opposed to the two times separately. This time-translation invariance allows the use of a forward time variable $t$ in Equations (1) and (2), representing the time lapsed since the injection of the initial neutron. The initial condition $P(0)=1$ for the single chain survival probability then corresponds to one initiating neutron at $t=0$ while $P_{E}(0)=1$ for the source extinction probability corresponds to the situation that no neutrons are present prior to the source being turned on.

\subsection{Analytic Solution}

In order to benchmark the numerical solutions of Equations (1) and (2) obtained in the following sections, we use a closed form analytic solution that can be readily obtained under the quadratic approximation (meaning $c h i_{\max }=2$ ) where the summations containing the factorial moments are truncated at order 2 Bell (1963); Prinja and Souto (2009). Equation (1) then reduces to a Bernoulli equation which can be transformed to a linear first order equation in the usual way and solved to obtain an exact solution for $P(t)$. This solution is then used in Equation $(2)$ to obtain $P_{E}(t)$. After some algebra, we obtain:

$$
\begin{aligned}
P(t) & =\frac{\exp (\alpha t)}{1+\frac{\chi_{2}^{\prime}}{2 \alpha}[\exp (\alpha t)-1]} \\
P_{E}(t) & =\left[1+\frac{\chi_{2}^{\prime}}{2 \alpha}(\exp (\alpha t)-1)\right]^{-\eta}
\end{aligned}
$$

where the parameters $\alpha, \chi_{2}^{\prime}$ and $\eta$ are defined as:

$$
\begin{aligned}
\alpha & =\frac{k_{\mathrm{eff}}-1}{\tau}, \\
\chi_{2}^{\prime} & =\frac{p_{f} \chi_{2}}{\tau}=\frac{\chi_{2} k_{\mathrm{eff}}}{\bar{\nu} \tau}, \\
\eta & =\frac{2 S_{0}}{\chi_{2}^{\prime}}=\left(\frac{2 \bar{\nu}}{\chi_{2}}\right) \frac{S_{0} \tau}{k_{\mathrm{eff}}} .
\end{aligned}
$$

We note that the above solutions are identical to those obtained by Bell using the quadratic approximation in the forward formulation Bell (1963); Prinja and Souto (2009). The solutions for the survival and source extinctions probabilities given by Equations(4) and (5) correspond to a supercritical system for which $k_{\text {eff }}>1$ or $\alpha>0$. 
For a subcritical system, so that $k_{\text {eff }}<1$, the above solutions are more conveniently expressed as:

$$
\begin{aligned}
P(t) & =\frac{\exp (-|\alpha| t)}{1+\frac{\chi_{2}^{\prime}}{2|\alpha|}[1-\exp (-|\alpha| t)]}, \\
P_{E}(t) & =\left[1+\frac{\chi_{2}^{\prime}}{2|\alpha|}(1-\exp (-|\alpha| t)]^{-\eta},\right.
\end{aligned}
$$

where $\alpha=-\left(1-k_{\text {eff }}\right) / \tau=-|\alpha|$, while for a critical system, i.e., $k_{\text {eff }}=1, \alpha=0$, Equations (4) and (5) reduce to:

$$
\begin{aligned}
P(t) & =\left[1+\frac{\chi_{2}}{2 \bar{\nu}} \frac{t}{\tau}\right]^{-1} \\
P_{E}(t) & =\left[1+\frac{\chi_{2}}{2 \bar{\nu}} \frac{t}{\tau}\right]^{-\eta}
\end{aligned}
$$

The quadratic approximation is known to be very accurate for systems that are not too far off critical and for $t \gg \tau$, and the fact that it enables exact solutions to be constructed makes this a useful model in general. We therefore use these solutions to guide the physical interpretation of the numerical solutions obtained below when all multiplicity terms are retained. To this end, it is helpful to consider the timeasymptotic limit as $t \rightarrow \infty$ of these solutions. For the single chain survival probability, this limit is given by:

$$
P(\infty)= \begin{cases}\frac{2 \bar{\nu}}{\chi_{2}}\left(\frac{k_{\mathrm{eff}}-1}{k_{\mathrm{eff}}}\right), & k_{\mathrm{eff}}>1 \\ 0, & k_{\mathrm{eff}}<1 \\ 0, & k_{\mathrm{eff}}=1\end{cases}
$$

The above result shows that for a critical or subcritical system the chain will eventually become extinct with probability one, while for a supercritical system there is a nonzero probability that the chain will not have died out. Under the latter conditions, it is known that the neutron population, if it has not become extinct, must necessarily be infinite, and $P(\infty)$ is then also referred to as the probability of divergence or the Probability of Initiation (POI) Bell (1963); Prinja and Souto (2009, 2010). Further noting that the factor $\frac{2 \bar{\nu}}{\chi_{2}}$ is nearly unity (see Table 1 below), we see that the POI is well approximated by the excess reactivity:

$$
P O I \approx \frac{k_{\mathrm{eff}}-1}{k_{\mathrm{eff}}} \approx k_{\mathrm{eff}}-1,
$$

with the second approximation holding for a weakly supercritical system $\left(k_{\text {eff }} \sim 1\right)$.

The corresponding $t \rightarrow \infty$ limits for the source extinction probability are given by:

$$
P_{E}(\infty)= \begin{cases}0, & k_{\text {eff }}>1 \\ {\left[1+\frac{\chi_{2}}{2 \bar{\nu}} \frac{k_{\mathrm{eff}}}{\left(1-k_{\mathrm{eff}}\right)}\right]^{-\eta},} & k_{\mathrm{eff}}<1 \\ 0 . & k_{\mathrm{eff}}=1\end{cases}
$$


This result shows that the neutron population can never become extinct for a critical or supercritical system in the presence of a steadily emitting random source, regardless of the source strength. However, there is a non-zero probability of the population becoming extinct if the system is subcritical, with this probability depending strongly on the source strength. The latter result simplifies to:

$$
P_{E}(\infty) \approx\left(1-k_{\mathrm{eff}}\right)^{S_{0} \tau}
$$

upon noting that $\eta \approx S_{0} \tau$ for a weakly subcritical system and again using the fact that $2 \bar{\nu} / \chi_{2} \approx 1$. Another useful result is given by $P_{E}(t)$ for $\alpha t \gg 1$, which can be inferred from Equation (5) to be:

$$
P_{E}(t) \approx\left(\frac{k_{\mathrm{eff}}}{k_{\mathrm{eff}}-1}\right)^{S_{0} \tau} \exp \left[-\left(k_{\mathrm{eff}}-1\right) S_{0} t\right] .
$$

For a fixed $k_{\text {eff }}$ this indicates that the time constant for the variation of the source extinction probability at late times is proportional to $1 / S_{0}$, the mean time between emission of source particles, and not the neutron lifetime $\tau$. Finally, the late time variation of source extinction probability for a critical system is readily seen from Equation (12) to be approximated by:

$$
P_{E}(t) \approx\left(\frac{\tau}{t}\right)^{S_{0} \tau}
$$

\subsection{Reference Parameters}

To numerically solve Equations(1) and (2) we use an algorithm found in Shampine and Gordon (1975) which employs adaptive time-stepping to allow the computationally efficient solution of these nonlinear first order ordinary differential equations. This algorithm has proved efficient, accurate and robust in this application.

The reference values for the parameters chosen to investigate the time variation of the survival and extinction probabilities, and relative to which uncertainty and sensitivity computations are performed, were obtained from the published literature. We use a value of 56.6 neutrons/s for the source strength $S_{0}$ and a neutron lifetime $\tau$ of $6.6 \times 10^{-9} \mathrm{~s}$, loosely based upon the GODIVA experiment (Orndoffl, 1957; Prinja and Souto, 2009)(REFs: Peterson,Orndoff,Prinja2008). The multiplicities were taken from Zucker and Holden (1986) and the factorial moments computed according to Equation (3). Both sets of data are tabulated below in Table 1 . The multiplication factor $k_{\text {eff }}$ was not treated as a random variable but rather was varied systematically. That is, the uncertainty and sensitivity analysis was carried out for fixed but different $k_{\text {eff }}$ values.

\section{Numerical Results for Reference Case}

In this section we present numerical results for $P(t)$ and $P_{E}(t)$ for the reference parameters given in Section 2.2 and consider $k_{\text {eff }}=0.99,1.01$ and 1.3, corresponding to a subcritical, weakly supercritical and strongly supercritical system, respectively. 


\begin{tabular}{|c|c|c|}
\hline$j$ & $p_{j}$ & $\chi_{j}$ \\
\hline 0 & 0.0319004 & 1 \\
\hline 1 & 0.1725213 & 2.4105 \\
\hline 2 & 0.3361397 & 4.6231 \\
\hline 3 & 0.3038798 & 6.7770 \\
\hline 4 & 0.1266155 & 7.2424 \\
\hline 5 & 0.0261843 & 5.3845 \\
\hline 6 & 0.0026170 & 2.6004 \\
\hline 7 & 0.0001421 & 0.7162 \\
\hline
\end{tabular}

Table 1: ${ }^{235} \mathrm{U}$ fission neutron multiplicities and factorial moments.

It is noted that the formulation of the point kinetics model in Equation which gives rise to the quantity $k_{\text {eff }}$ is based on the assumption the system is near critical and, in the case where $k_{\text {eff }}=1.3$, this approximation is not strictly applicable. It is to be expected that this may introduce some error if the simulated results were compared to experimental results. A full extension of the point kinetics model to a greater range of reactivities is beyond the scope of this study so we use this standard formulation and so, despite this limitation, this case is included in order to illustrate informative qualitative behaviour.

As mentioned earlier, in addition to establishing the robustness and accuracy of the deterministic solver, the numerical results displayed here helps develop a qualitative feel for the dependence of the solution on the different parameters and aid in the interpretation of the results when uncertainty is introduced into the parameters. The numerical and analytical results under the quadratic approximation are displayed in Figure 1, showing that the simulated results match the analytic results to a high degree of accuracy and verifying the model is indeed solving Equations (1) and (2) accurately. The numerical results confirm our earlier conclusions based on the analytic solution that a single initiating neutron and its progeny will become extinct with certainty unless the system is supercritical. In the presence of a random intrinsic source, on the other hand, the population diverges with certainty unless the system is subcritical or critical.

\subsection{Base Line Results}

We now consider the numerical solutions in the general case, with $\chi_{\max }$ set equal to 7 . The results are displayed in Figure 2. As can be seen, the survival chance of a neutron chain is initially 1 before decreasing on a timescale comparable to a few neutron lifetime $\tau\left(6.6 \times 10^{-9} \mathrm{~s}\right)$. For the subcritical case $\left(k_{\mathrm{eff}}=0.99\right)$ the time-asymptotic survival probability, i.e., the POI, approaches 0 . For the slightly supercritical case $\left(k_{\text {eff }}=1.01\right)$ it tends towards a steady value of approximately 0.01038 , which is very close to the analytic value of 0.010325 obtained using Equation (13) for the quadratic approximation. The high supercritical case $\left(k_{\text {eff }}=1.3\right)$ tends towards a POI of 0.275 , while the quadratic approximation yields 0.24065 , a relatively small $12.5 \%$ deviation from the exact value for a highly reactive system. 


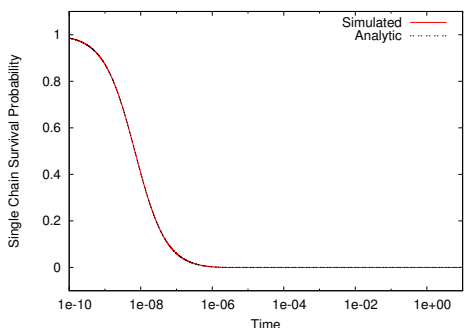

(a) $k_{\text {eff }}=0.99$

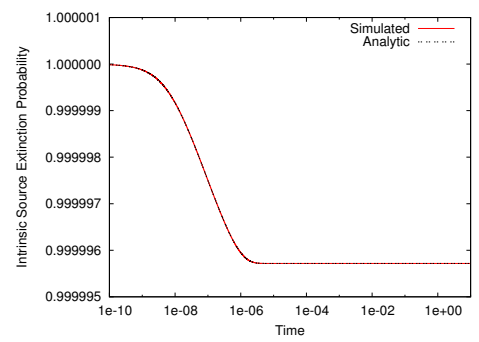

(d) $k_{\text {eff }}=0.99$

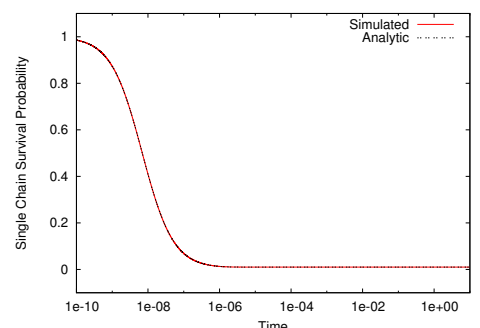

(b) $k_{\text {eff }}=1.01$

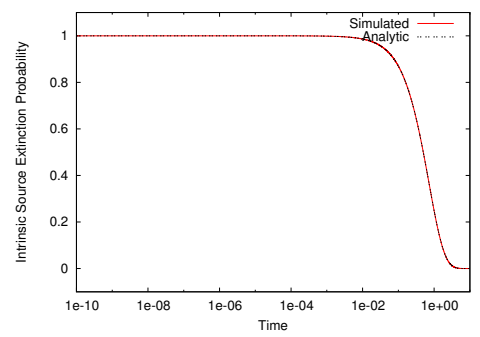

(e) $k_{\text {eff }}=1.01$

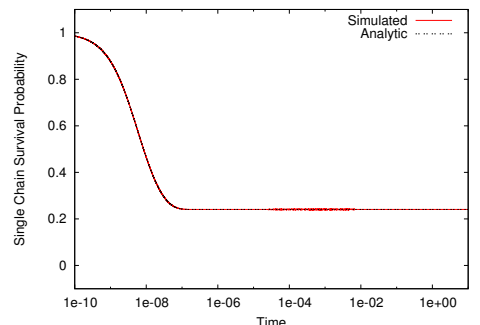

(c) $k_{\text {eff }}=1.3$

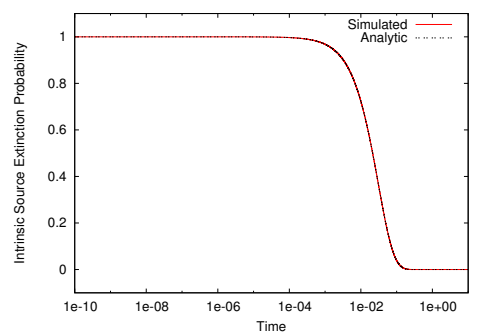

(f) $k_{\text {eff }}=1.3$

Figure 1: Comparison of the solution of the model's results with $\chi_{\max }=2$ to the analytical results of Equations (4) and (5).

In the presence of a random source, the probability of no neutrons being present at a given time is 1 at $t=0$ and tends towards 0 with increasing time for all supercritical cases. That is, the population always diverges in a supercritical (as well as critical) system. For the subcritical case $\left(k_{\text {eff }}=0.99\right)$, however, the probability approaches the non-zero limiting value of 0.9999961 . This is very close to one because neutrons and any neutron chain which may ensue only survive in the system for an average of $\sim 1 \times 10^{-7}$ s and only 56 are injected each second meaning the odds of there being a non-zero number of neutrons in the system is very small at any given time. We note that the corresponding exact result under the quadratic approximation obtained from Equation (15) is 0.9999981, which is an extremely accurate value when compared to the exact numerical result.

Before proceeding with the stochastic uncertainty analysis, we first consider the effects of systematic changes in the system parameters on the single chain survival probability and the source extinction probability.

\subsection{Variation of $k_{\mathrm{eff}}$}

We first examine the POI, i.e., the asymptotic value of the single chain neutron survival probability, as a function of $k_{\text {eff }}$. Numerical results are obtained for values of $k_{\text {eff }}$ between 1 and 2.4105, the upper bound corresponding to $k_{\infty}$, and the survival probability is recorded at 10 s by which time it has asymptoted to the POI. The numerical results as well as the exact result from the quadratic approximation given by Equation (13) are shown in Figure 3. It is observed that the quadratic approximation is accurate to within $0.33 \%$ for $k_{\text {eff }}<1.1$ over which range the numerical results scale linearly with $k_{\text {eff }}-1$, but for larger of $k_{\text {eff }}$ it increasingly deviates from the exact value. 


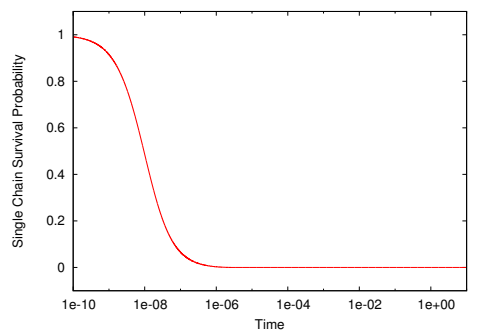

(a) $k_{\text {eff }}=0.99$

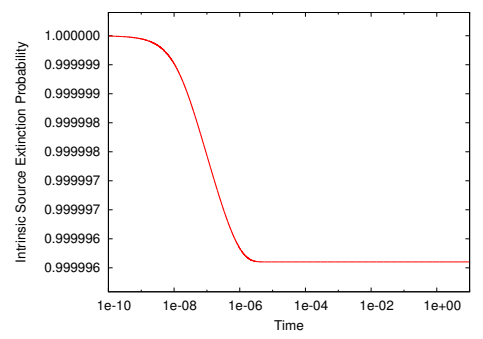

(d) $k_{\text {eff }}=0.99$

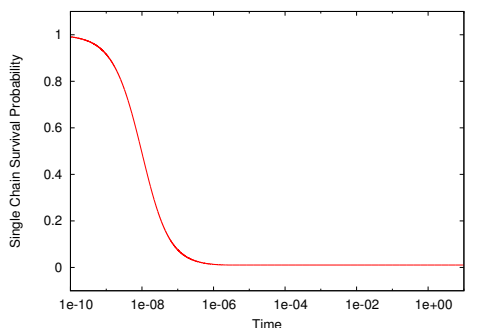

(b) $k_{\text {eff }}=1.01$

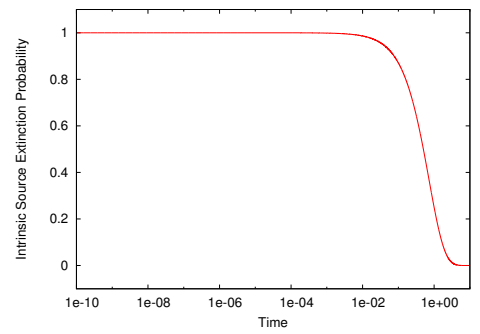

(e) $k_{\text {eff }}=1.01$

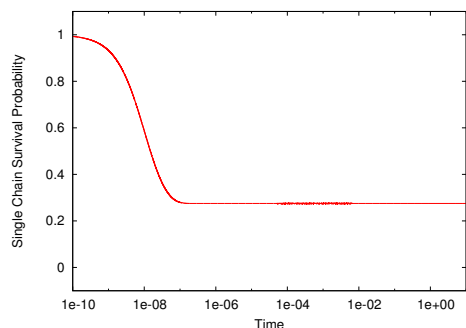

(c) $k_{\text {eff }}=1.3$

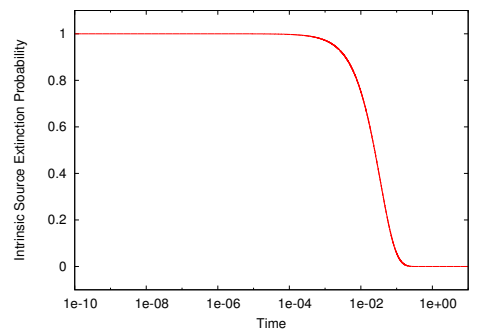

(f) $k_{\text {eff }}=1.3$

Figure 2: The time-dependent single neutron chain and neutron extinction probability.

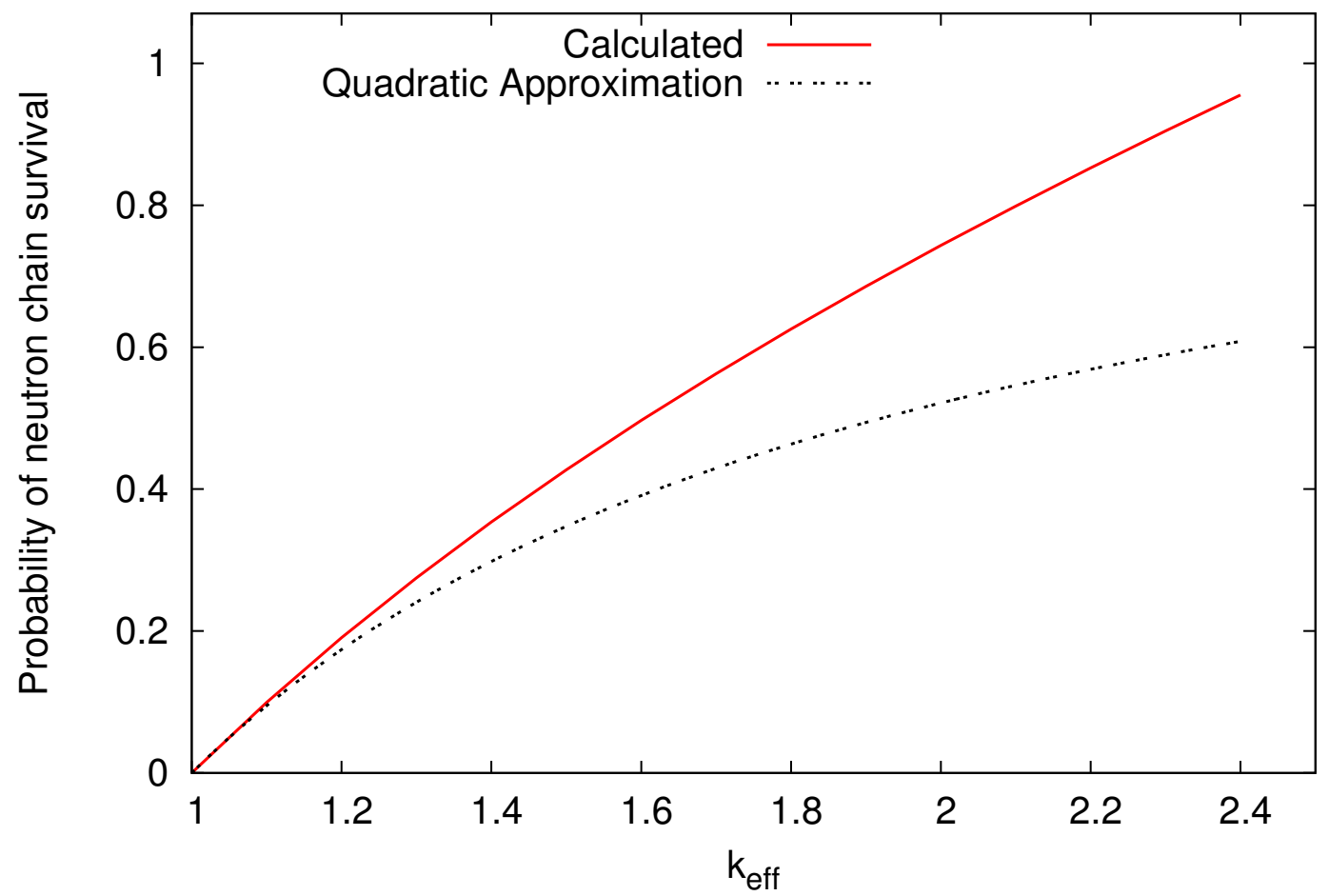

Figure 3: Probability of initiation as a function of $k_{\mathrm{eff}}$. The calculated values are produced by this code with $\chi_{\max }=7$ whilst the quadratic approximation is calculated by Equation (13).

\subsection{Variation of $\chi_{\max }$}

When comparing the time dependent analytical solutions in Figure 1 obtained by setting $\chi_{\max }=2$ to the numerical results in Figure 2 for the more general case corresponding to $\chi_{\max }=7$, they do not appear markedly different. However, in Figure 
3 a difference can be seen between the analytical $\left(\chi_{\max }=2\right.$ case $)$ and the simulated case with $c h i_{\max }=7$. In Figure 4 we present a systematic variation of the value of the value of $\chi_{\max }$ over a broader variation of $\chi_{\max }$ values for three systems with different criticalities.

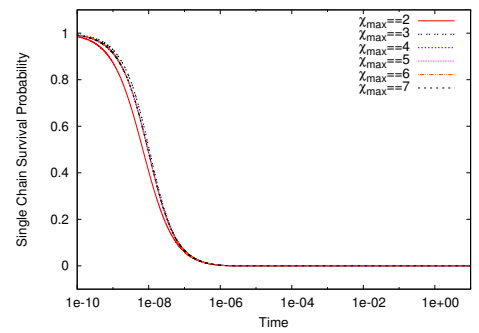

(a) $k_{\text {eff }}=0.99$

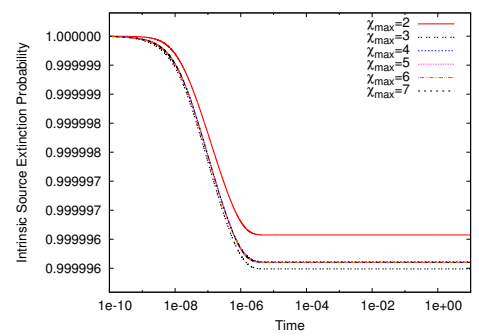

(d) $k_{\text {eff }}=0.99$

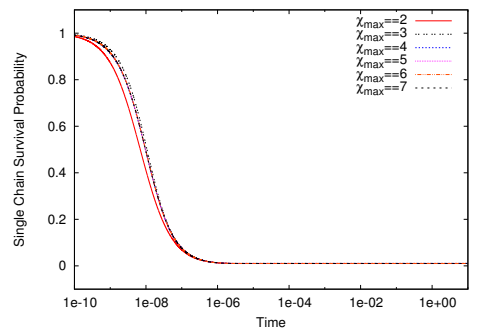

(b) $k_{\text {eff }}=1.01$

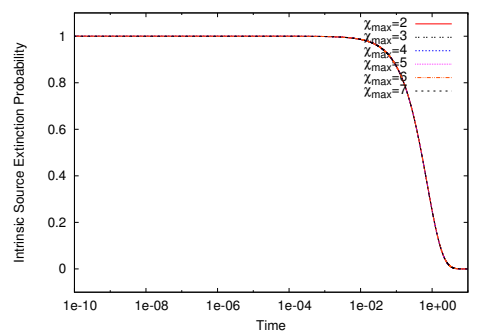

(e) $k_{\text {eff }}=1.01$

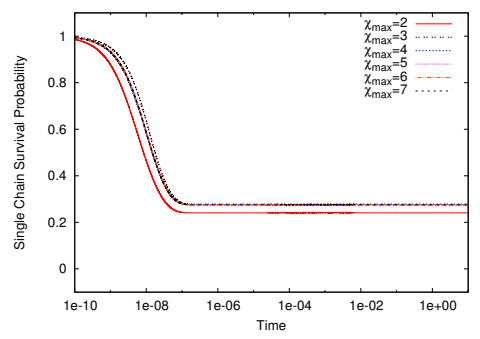

(c) $k_{\text {eff }}=1.3$

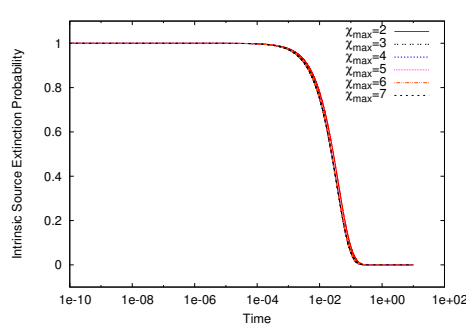

(f) $k_{\text {eff }}=1.3$

Figure 4: The time-dependent single neutron chain and neutron extinction probability for various values of $\chi_{\max }$.

As can be seen, regardless of the value of $\chi_{\max }$ used the results obtained are qualitatively similar. The final value of the single chain survival probability tends to zero for all values of $\chi_{\max }$ and the extinction probability tends to $1-3.43 \times 10^{-6}$ for the case where $\chi_{\max }=2$ and $1-3.90 \times 10^{-6}$ for the case where $\chi_{\max }=7$. For both supercritical cases the extinction probability tends to zero for all values of $\chi_{\max }$. For the weakly supercritical case the survival probability tends to $1.032 \times 10^{-2}$ for the case where $\chi_{\max }=2$ and $1.038 \times 10^{-2}$ for the case where $\chi_{\max }=7$. For the highly supercritical case the survival probability tends to 0.241 for the case where $\chi_{\max }=2$ and 0.275 for the case where $\chi_{\max }=7$. This continues the trend observed in Figure 3 where the results of the case $\chi_{\max }=2$ and $\chi_{\max }=7$ are more different for higher values of $k_{\text {eff }}$.

\subsection{Variation of source strength}

We consider next the effect of varying the intensity of the intrinsic neutron source on the extinction probability $P_{E}(t)$. We plot $P_{E}(t)$ in Figure 5 for several values of $S_{0}$ for the three cases of $k_{\text {eff }}$.

The extinction probability clearly shows a strong dependence on the source strength. In the strongly supercritical case $\left(k_{\text {eff }}=1.3\right)$ the extinction possibility approaches 0 at different rates depending on the source strength. We may note that the majority of the drop from 1 to 0 occurs at a timescale of the order of $\frac{1}{S_{O}}$. That is because for a highly reactive system, there is a high probability of chains being sustained and hence a 


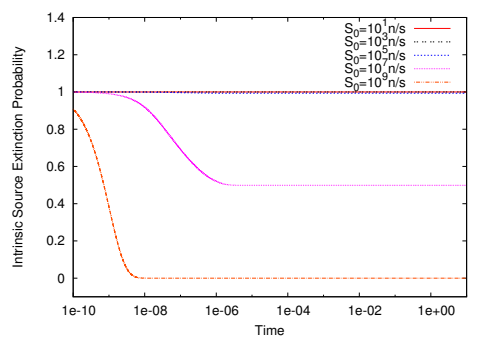

(a) $k_{\text {eff }}=0.99$

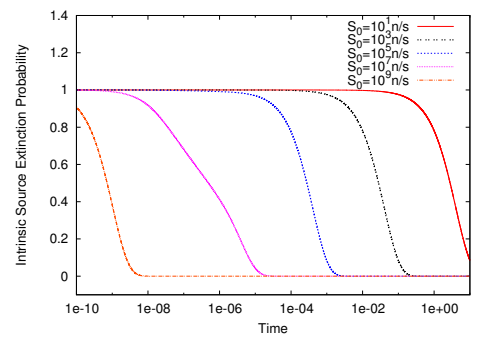

(b) $k_{\text {eff }}=1.01$

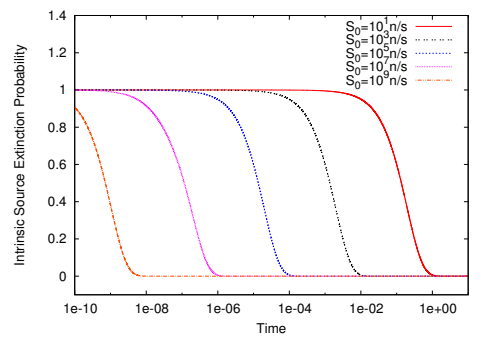

(c) $k_{\text {eff }}=1.3$

Figure 5: The time-dependent neutron extinction probability for various values of source strength.

low probability of extinction. This observation is consistent with the analytic solution given by Equation (17) which shows that for $k_{\text {eff }}$ not close to unity, the time scale is determined by the inverse of the source strength.

For the weakly supercritical case $\left(k_{\text {eff }}=1.01\right)$ the same qualitative pattern is true - the higher the source strength the quicker the extinction probability drops to zero. However, this time the timescale is approximately equal to $\frac{100}{S_{O}}$. This is because each neutron only has $\sim\left(k_{\text {eff }}-1\right)=0.01$ chance of initiating a chain reaction which does not die out so it takes more injected neutrons before there is a large chance of one having initiated a sustained reaction. This gives a time constant of $1 /\left[S_{0}\left(k_{\mathrm{eff}}-1\right)\right]$, again consistent with the analytical solution, Equation (17). In the subcritical case, although the extinction probability reduces with increasing source strength, the probability of extinction remains non-zero, confirming the analytical solution given by Equation (16). This is in contrast to the supercritical cases where the population will ultimately diverge.

\section{Modelling Parameter Uncertainty}

As discussed in Section 1, the model parameters are not known with precision due to uncertainties in the data and the use of a lumped representation of the physical system as discussed in Section 1. To evaluate the system response to uncertainty it is necessary to (i) characterize the uncertainty in the input parameters, (ii) develop a method to propagate the uncertainty through the model equations, and (iii) have a framework for quantifying the resulting variability in the output variables, in our case the single chain survival probability $P(t)$ and the extinction probability in the presence of an intrinsic random source $P_{E}(t)$. In this section and the next, we describe the application of Monte Carlo and stochastic spectral techniques to uncertainty propagation and quantification in the problem of interest here.

Irreducible or aleatoric uncertainty, as input parameter uncertainty is commonly referred to, is traditionally described using probabilistic formulations in which the uncertainty is characterized by suitable PDFs. As these distributions are not known in advance, it is a customary procedure to implement several PDFs (uniform, normal, lognormal, etc.) that have the same mean and variance, as well as covariance if the 
parameters are correlated. In this work, however, we consider only the uniform distribution and moreover assume all parameters are statistically independent, deferring investigation of more complex characterizations of input variable uncertainty to future work. Thus, the random variation of an arbitrary model parameter $\alpha_{i}$ is bounded between the limits $\alpha_{i, \min }$ and $\alpha_{i, \max }$ and the distribution of each $\alpha_{i}$ is mapped to a standard uniform random variable $\xi_{i}$ with $\mathrm{PDF}$ :

$$
p_{\xi_{i}}\left(\xi_{i}\right) d \xi_{i}=\frac{d \xi_{i}}{2}, \quad \xi_{i} \in[-1,1] .
$$

The mapping is given by the following statement of conservation of probability:

$$
\begin{aligned}
\int_{-1}^{\xi_{i}} p_{\xi_{i}}\left(\xi_{i}^{\prime}\right) d \xi_{i}^{\prime} & =\int_{-1}^{\alpha_{i}} p_{\alpha_{i}}\left(\alpha_{i}^{\prime}\right) d \alpha_{i}^{\prime} \\
\Rightarrow \quad \int_{-1}^{\xi_{i}} \frac{d \xi_{i}}{2} & =\int_{\alpha_{i, \min }}^{\alpha_{i}} \frac{d \alpha_{i}}{\alpha_{i, \max }-\alpha_{i, \min }}
\end{aligned}
$$

and, for a given realization which we denote by the label $\omega$, this yields the relationship:

$$
\alpha_{i}(\omega)=\overline{\alpha_{i}}+\sqrt{3} \sigma_{\alpha_{i}} \xi(\omega)
$$

From these results it readily follows that:

$$
\alpha_{i, \min }=\overline{\alpha_{i}}-\sqrt{3} \sigma_{\alpha_{i}} \quad \text { and } \quad \alpha_{i, \max }=\overline{\alpha_{i}}+\sqrt{3} \sigma_{\alpha_{i}}
$$

where $\overline{\alpha_{i}}$ is the mean and $\sigma_{\alpha_{i}}$ is the standard deviation for the input parameter $\alpha_{i}$.

In the ensuing, the factorial moments $\chi_{i}$, the neutron lifetime $\tau$, and intrinsic source strength $S_{0}$ are taken to be independent and uniformly distributed parameters, each with a mean value set equal to the corresponding reference value given in the previous section, and a variance that must be prescribed. The chain survival and source extinction probabilities are now time dependent stochastic processes, i.e., they are random variables indexed by the time variable, and realizations of these probabilities are obtained by solving Equations (1) and (2) for different realizations of the input parameters. We emphasize the fact that these equations are now random differential equations by writing them as:

$$
\begin{aligned}
\frac{\partial P(t, \omega)}{\partial t} & =\left[\frac{k_{\mathrm{eff}}-1}{\tau(\omega)}\right] P(t, \omega)-\frac{p_{f}}{\tau(\omega)} \sum_{i=2}^{\chi_{\max }}(-1)^{i} \frac{\chi_{i}(\omega)}{i !} P^{i}(t, \omega), \quad P(0)=1, \\
\frac{\partial P_{E}(t, \omega)}{\partial t} & =S_{0}(\omega)\left[\sum_{i=1}^{\chi_{\max }}(-1)^{i} \frac{\chi_{i}(\omega)}{i !} P^{i}(t, \omega)\right] P_{E}(t, \omega), \quad P_{E}(0)=1,
\end{aligned}
$$

where all realizations are assumed to have the same initial value. Thus, propagation of uncertainty reduces to solving the above equations for a sufficiently large number of realizations of the input parameters drawn from their specified PDFs to 
create an ensemble of outputs, where each member of the ensemble consists of the pair of probabilities as a function of time. This is followed by statistical processing of this ensemble to extract usable quantitative information. Here we are specifically interested in the mean and variance of the survival and extinction probabilities as a function of time, as well as PDFs of these quantities at selected time instants. This random sampling or Monte Carlo approach is widely employed for aleatoric UQ computations as it is nonintrusive and hence very straightforward to implement. Besides often being the only viable method, especially for problems with very large numbers of variable inputs, it is commonly used to benchmark approximate but computationally more efficient methods.

Given realizations of a model output $\mathcal{J}(\omega)$ generated using this random sampling approach, the mean and variance can be computed as:

$$
\begin{aligned}
\overline{\mathcal{J}} & =\frac{1}{N} \sum_{i=1}^{N} \mathcal{J}\left(\omega_{i}\right), \\
\sigma_{\mathcal{J}}^{2} & =\frac{1}{N} \sum_{i=1}^{N}\left(\mathcal{J}\left(\omega_{i}\right)-\overline{\mathcal{J}}\right)^{2},
\end{aligned}
$$

and the PDFs are constructed by accumulating contributions from all realizations on an appropriate grid of the chain survival and source extinction probability values. We note that the asymptotic numerical error of the Monte Carlo method scales as approximately $\mathcal{O}\left(N^{-\frac{1}{2}}\right)$ where $N$ is the number of realizations of the deterministic system, so that a sufficiently large ensemble must be computed in order to achieve reasonable statistics. For this reason, alternative UQ techniques that converge faster than the brute force random sampling approach presented above have been developed and are gaining considerable traction. We will describe one such alternate in the next section, which will also constitute a key contribution of this article. However, we first show that the PDFs of the neutron probabilities of interest can be obtained exactly under the quadratic approximation and assumed uniform distribution of input variable PDFs. These exact solutions prove invaluable in establishing numerical convergence rates with respect to the random dimension $\omega$ of both the Monte Carlo and the stochastic spectral UQ methods employed in this work.

\subsection{Exact Distribution of Single Chain Survival Probability in the Quadratic Approx- imation}

For each realization of the input variables, the survival and extinction probabilities can be computed exactly when the quadratic approximation is used, as was demonstrated in the previous section. In the case of the single chain survival probability, this explicit solution can be combined with probability transformation rules to obtain the PDFs of the two chain probabilities as a function of time when $\chi_{2}$ only has an associated uncertainty, as we demonstrate next. 
Recalling these solutions but now recognizing that the solutions represent individual realizations, we write them as:

$$
\begin{aligned}
P(t, \omega) & =\frac{\exp (\alpha(\omega) t)}{1+\frac{\chi_{2}^{\prime}(\omega)}{2 \alpha(\omega)}[\exp (\alpha(\omega) t)-1]}, \\
P_{E}(t, \omega) & =\left[1+\frac{\chi_{2}^{\prime}(\omega)}{2 \alpha(\omega)}(\exp (\alpha(\omega) t)-1)\right]^{-\eta(\omega)},
\end{aligned}
$$

For a given realization $\omega$ of the random parameters, the above solutions are deterministic functions of time but at a fixed time these solutions are random variables. The PDFs of the survival and extinction probabilities can be constructed at any given time using the following transformation rule. Given a random scalar $y(\omega)$ that is a function of a finite number of random parameters $\vec{\xi}(\omega)=\left\{\xi_{1}(\omega), \xi_{2}(\omega) \cdots \xi_{N}(\omega)\right\}$ and a nonrandom real parameter $t$ through the mapping:

$$
y(\omega, t)=g[t, \vec{\xi}(\omega)]
$$

the PDF of $y$ is given by:

$$
p_{y}(y, t)=\int_{\mathcal{D}_{\mathcal{N}}} \delta[y-g(t, \vec{\xi})] p_{\xi}(\vec{\xi}) d^{N} \xi,
$$

where $p_{\xi}(\vec{\xi})$ is the joint distribution of the $N$ random parameters and $\mathcal{D}_{\mathcal{N}}$ is the $N$ dimensional support. When the parameters are statistically independent, as is the case here, the joint distribution factorizes into a product of the individual PDFs. Consider the case when only the multiplicity moment $\chi_{2}$ is random in single chain survival probability given by Equation (26). Then, the PDF may be written as:

$$
p_{s}(s, t)=\int_{\chi_{2, \min }}^{\chi_{2, \max }} \delta\left[s-\frac{\exp \alpha t}{1+\chi_{2} \gamma(t)}\right] p_{\chi_{2}}\left(\chi_{2}\right) d \chi_{2}
$$

where $s$ is a particular value of the single chain survival probability and

$$
\gamma(t)=\frac{k}{2 \bar{\nu}(k-1)}[\exp (\alpha t)-1]
$$

Reverting to the standard uniformly distributed random variable $\xi$, Equation (30) becomes:

$$
p_{s}(s, t)=\int_{-1}^{1} \delta\left[s-\frac{\exp \alpha t}{1+\gamma(t)\left(\overline{\chi_{2}}+\sqrt{3} \sigma_{\chi_{2}} \xi\right)}\right] \frac{d \xi}{2} .
$$

Now changing the independent variable from $\xi$ to

$$
y=s\left[1+\gamma(t)\left(\overline{\chi_{2}}+\sqrt{3} \sigma_{\chi_{2}} \xi\right)\right]-\exp (\alpha t),
$$


and using the delta function identity $\delta[a x]=(1 /|a|) \delta[x]$, Equation (32) may be simplified to yield:

$$
p_{s}(s, t)=\frac{1}{2 \sqrt{3} \gamma(t) \sigma_{\chi_{2}} s^{2}} \int_{y_{\min }}^{y_{\max }} \delta(y)[y+\exp (\alpha t)] d y
$$

where the integration limits are given by:

$$
\begin{aligned}
y_{\text {min }} & =s\left[1+\gamma(t)\left(\overline{\chi_{2}}-\sqrt{3} \sigma_{\chi_{2}}\right)\right]-\exp (\alpha t) \\
& =s\left[1+\gamma(t) \chi_{2, \min }\right]-\exp (\alpha t), \\
y_{\max } & =s\left[1+\gamma(t)\left(\overline{\chi_{2}}+\sqrt{3} \sigma_{\chi_{2}}\right)\right]-\exp (\alpha t) \\
& =s\left[1+\gamma(t) \chi_{2, \max }\right]-\exp (\alpha t) .
\end{aligned}
$$

The integral will give a non-zero value only if $y_{\min }<0$ and $y_{\max }>0$, and the final result may then be expressed as:

$$
p_{s}(s, t)= \begin{cases}\frac{\exp (\alpha t)}{2 \sqrt{3} \gamma(t) \sigma_{\chi_{2}} s^{2}}, & \frac{\exp (\alpha t)}{1+\gamma(t) \chi_{2, \max }}<s<\frac{\exp (\alpha t)}{1+\gamma(t) \chi_{2, \min }} \\ 0 & \text { otherwise }\end{cases}
$$

where $\chi_{2, \min }$ and $\chi_{2, \max }$ are defined in Equation (35) and Equation (36) respectively. It is readily shown that $\int_{s_{\min }}^{s_{\max }} p_{s}(s, t) d s=1$, where $s_{\min }$ and $s_{\max }$ are defined by the lower and upper bounds on $s$ given in Equation (37), and hence $p_{s}(s, t)$ is a true PDF.

Also useful for benchmarking the numerical solutions are the mean and variance of the single chain survival probability, which is readily obtained from the exact distributions:

$$
\begin{aligned}
\bar{s}(t) & =\int_{s_{\min }}^{s_{\max }} s p_{s}(s, t) d s \\
& =\frac{\exp (\alpha t)}{2 \sqrt{3} \sigma_{\chi_{2}} \gamma(t)} \ln \left[\frac{1+\chi_{2, \max } \gamma(t)}{1+\chi_{2, \min } \gamma(t)}\right], \\
\sigma_{s}^{2}(t) & =\int_{s_{\min }}^{s_{\max }} s^{2} p_{s}(s, t) d s-\bar{s}^{2}(t) \\
& =\frac{\exp (2 \alpha t)}{\left[1+\chi_{2, \min } \gamma(t)\right]\left[1+\chi_{2, \max } \gamma(t)\right]}-\bar{s}^{2}(t) .
\end{aligned}
$$

Exact output distributions corresponding to random variation of other input parameters can be similarly derived.

\section{Generalised Polynomial Chaos (GPC)}

An alternative approach to the Monte Carlo method for uncertainty quantification is the so called spectral stochastic expansion technique. This class of method represents the response (and/or inputs) in a suitable function space spanned by a polynomial 
basis (Spanos and Ghanem, 1989). Each of the polynomials, which are known as a polynomial chaos, are orthogonal with respect to the joint distribution of the input variables and form a complete set. The response function is then approximated using a truncated expansion of the polynomial chaoses. Once the coefficients of the expansion are computed, they may be used to calculate the statistics of the response function. The maximum number of coefficients in the expansion is a function of the number of dimensions of the response surface, $M$, and the maximum polynomial order $p$. Depending upon the manner in which the polynomial expansion is built, the number of coefficients can increase exponentially with $M$ and $p$. This is known as the curse of dimensionality.

In the original work by Wiener (Wiener, 1938) Gaussian random variables were used with a Hermite polynomial basis to describe the model response. This so called WienerChaos expansion can approximate any functional in the continuously differentiable $L_{2}$ space and converges in the $L_{2}$ sense (Cameron and Martin, 1947). In practice, any statistical distribution can be associated with an orthogonal polynomial (Xiu and Karniadakis, 2002). This generalisation of Wiener-Chaos is known as Generalised Polynomial Chaos (GPC). In this work the uncertainties are defined on the unit cube $\Omega^{M}=[-1,1]^{M}$ for which the appropriate basis functions are the Legendre polynomials. The model response, as a function of time $t$, is represented as follows:

$$
\begin{aligned}
\mathrm{X}(t, \boldsymbol{\xi}) & =\mathrm{x}_{0}(t) \Gamma_{0}+\sum_{i_{1}=1}^{\infty} \mathrm{x}_{i_{1}}(t) \Gamma_{1}\left(\xi_{i_{1}}\right) \\
& +\sum_{i_{1}=1}^{\infty} \sum_{i_{2}=1}^{i_{1}} \mathrm{x}_{i_{1} i_{2}}(t) \Gamma_{2}\left(\xi_{i_{1}}, \xi_{i_{2}}\right) \\
& +\sum_{i_{1}=1}^{\infty} \sum_{i_{2}=1}^{i_{1}} \sum_{i_{3}=1}^{i_{2}} \mathrm{x}_{i_{1} i_{2} i_{3}}(t) \Gamma_{3}\left(\xi_{i_{1}}, \xi_{i_{2}}, \xi_{i_{3}}\right) \\
& +\cdots,
\end{aligned}
$$

or more compactly:

$$
\mathrm{X}(t, \boldsymbol{\xi})=\sum_{\boldsymbol{\alpha} \in \mathcal{A}^{M}} \mathrm{x}_{\boldsymbol{\alpha}}(t) \Phi_{\boldsymbol{\alpha}}(\boldsymbol{\xi}) \quad \boldsymbol{\xi} \in[-1,1]^{M}
$$

where $\boldsymbol{\xi}=\left(\xi_{i_{1}}, \cdots, \xi_{i_{n}}\right)$ is a vector of independent, identically distributed standard uniform variables and $\mathcal{A}^{M}$ is the index set which is defined as $\mathcal{A}^{M} \equiv\left\{\boldsymbol{\alpha} \in \mathbb{N}^{M}\right\}$ where $M$ is the number of dimensions. The subscript $\boldsymbol{\alpha}$ is an index that denotes the orders of the univariate Legendre polynomials in the product

$$
\Phi_{\boldsymbol{\alpha}}(\boldsymbol{\xi})=\prod_{i=1}^{M} L_{\alpha_{i}}\left(\xi_{i}\right)
$$


The polynomial chaos basis functions are orthogonal which implies

$$
\left\langle\Phi_{\alpha}, \Phi_{\beta}\right\rangle \equiv \int_{\Omega^{M}} \Phi_{\alpha}(\boldsymbol{\xi}) \Phi_{\beta}(\boldsymbol{\xi}) p(\boldsymbol{\xi}) d \boldsymbol{\xi}=\prod_{i=1}^{M} N_{i}^{2} \delta_{i j},
$$

where $\delta_{i j}$ is the Kronecker delta and $N_{i}^{2}$ is a normalisation constant which, for the Legendre polynomials, is given by

$$
N_{i}=\frac{1}{2 i+1}
$$

Naturally, for the purposes of numerical computation, the series given by Equation (41) must be truncated. The most commonly used approach is to restrict the maximum total order of the multivariate polynomial basis to $\leq p$. This imposes the following restriction on the index sets

$$
\mathcal{A}^{M, p} \equiv\left\{\boldsymbol{\alpha} \in \mathbb{N}^{M}:\|\boldsymbol{\alpha}\|_{1} \leq p\right\} \quad ; \quad\|\boldsymbol{\alpha}\|_{1} \equiv \sum_{i=1}^{M} \alpha_{i}
$$

For a number of random dimensions $M$ and maximum polynomial order $p$ the total number of coefficients in the expansion is given by:

$$
N_{P}+1=\frac{(M+p) !}{M ! p !}=\left(\begin{array}{c}
M+p \\
M
\end{array}\right) .
$$

Equation (41) can now be written as

$$
\mathrm{X}(t, \boldsymbol{\xi})=\sum_{\boldsymbol{\alpha} \in \mathcal{A}^{M, p}} \mathrm{x}_{\boldsymbol{\alpha}}(t) \Phi_{\boldsymbol{\alpha}}(\boldsymbol{\xi})
$$

The statistics of the response function can be calculated from the coefficients of the expansion in (46). Expressions for the mean and variance are

$$
\begin{aligned}
\overline{\mathrm{X}}(t) & =\mathrm{x}_{0}(t), \\
\sigma_{\mathrm{X}}^{2} & =\sum_{\boldsymbol{\alpha} \in \mathcal{A}^{M, p}, \boldsymbol{\alpha} \neq \mathbf{0}} \mathrm{x}_{\boldsymbol{\alpha}}^{2}(t) \Phi_{\boldsymbol{\alpha}}(\boldsymbol{\xi}) .
\end{aligned}
$$

There are two broad approaches to calculating these coefficients: the intrusive approach and the non-intrusive approach. The intrusive method replaces the response function in the model directly with the expansion in Equation (46)). A Galerkin projection is then used to produce a set of coupled equations for the GPC coefficients, see (Ghanem, 1999; Stefanou, 2009) for examples. This method provides accurate solutions for the GPC coefficients but does become computationally demanding with large $M$ or $p$ due to the rapid increase in the number of unknowns described in Equation 
(45). The intrusive method also requires the complete reformulation of any existing code which is often difficult, if not impossible. Therefore, non-intrusive approaches based on projection (Xiu and Hesthaven, 2005) have been proposed which calculate the GPC coefficients via the following integral

$$
\mathrm{x}_{\boldsymbol{\alpha}}(t)=\frac{\int \Phi_{\boldsymbol{\alpha}}(\boldsymbol{\xi}) \mathrm{X}(t, \boldsymbol{\xi}) d \boldsymbol{\xi}}{\int \Phi_{\boldsymbol{\alpha}}^{2}(\boldsymbol{\xi}) d \boldsymbol{\xi}},
$$

which is calculated using a quadrature scheme. Thus its implementation is similar to that of Monte Carlo, where the deterministic model is solved repetitively for a number of discrete points in the random space.

\subsection{Non-intrusive Sparse Grid Quadrature Methods}

The multidimensional integral in the numerator of Equation (49), which is of the form

$$
\boldsymbol{I} f=\int_{[-1,1]^{M}} f(\boldsymbol{\xi}) d \boldsymbol{\xi}
$$

can be costly to evaluate, particularly if the number of dimensions $M$ is large. One approach which has proven successful (Gerstner and Griebel, 1998; Nobile et al., 2008 ) is the quadrature method. In the univariate case, a quadrature rule $U_{l}$ of level $l$ approximates an integral using a weighted sum of function evaluations by the following expression:

$$
U_{l} f \approx \sum_{i=1}^{n_{l}} w_{i} f\left(\xi_{i}\right)
$$

The immediate approach to the integral in Equation (50) is to take a tensor product of the univariate rules in Equation (51) yielding the following expression:

$$
\boldsymbol{I} f \approx \sum_{i_{1}=1}^{n_{l_{1}}} \cdots \sum_{i_{M}=1}^{n_{l_{M}}} w_{i_{1}} \cdots w_{i_{m}} f\left(\xi_{i_{1}} \cdots \xi_{i_{M}}\right) .
$$

However, this leads to an exponential dependence of the number of points on the dimension $M$. This exponential dependence has been alleviated to some extent by sparse-grid quadrature schemes. The Smolyak construction (Smolyak, 1963) for sparse grids are built upon tensor products of the following hierarchical difference sets:

$$
\Delta_{k}:=U_{m}-U_{m-1} \text { with } U_{0}=0,
$$

for $k \geq 1$. The univariate quadrature rule in Equation (51) can then be built from a summation of these difference sets, namely 


$$
U_{l}=\sum_{k=1}^{l} \Delta_{m}
$$

The Smolyak construction $A(\mathcal{L}, M)$ for a $\mathrm{M}$ dimensional quadrature rule of total level $\mathcal{L}$ is

$$
A(\mathcal{L}, M)=\sum_{|\boldsymbol{k}|_{1} \leq M+\mathcal{L}-1} \bigotimes_{i=1}^{M} \Delta_{k_{i}}
$$

where $|\boldsymbol{k}|_{1}=\sum_{i=1}^{M} k_{i}$ is the $L_{1}$ norm. Thus, out of all the possible sets $\boldsymbol{k} \in \mathbb{N}$, only those are chosen whose 1-norm is smaller than a constant. If, instead, we use the $|\boldsymbol{k}|_{\infty}$ norm

$$
A(\mathcal{L}, M)=\sum_{k_{1}=1}^{\mathcal{L}} \cdots \sum_{k_{M}=1}^{\mathcal{L}} \Delta_{k_{1}} \otimes \cdots \otimes \Delta_{k_{M}}
$$

we recover the tensor product formula Equation (52) where the order for each dimension is equal to $\mathcal{L}$. For numerical purposes it is more convenient to rewrite Equation (55) in terms of the univariate rules $U_{m_{k}}$. The tensor product of the difference sets $\Delta_{\boldsymbol{k}}$ for an index set $\boldsymbol{k}$ is given by (Wasilkowski and Wozniakowski, 1995):

$$
A(l, M) f=\sum_{M \leq|\boldsymbol{k}|_{1} \leq \mathcal{L}+M-1}(-1)^{M+\mathcal{L}-1-|\boldsymbol{k}|}\left(\begin{array}{c}
M-1 \\
|\boldsymbol{k}|-\mathcal{L}
\end{array}\right)\left(\bigotimes_{i=1}^{M} U_{k_{i}}\right) f .
$$

For integrals defined on the domain $[-1,1]$, a Gauss-Legendre quadrature is used to construct the Smolyak sparse grids as it provides the optimal order of accuracy. A comparison between a tensor product and Smolyak grid is shown in Fig.(6) for two dimensions and a level $\mathcal{L}=3$ quadrature rule.

\subsection{Global Sensitivity Analysis}

Sensitivity analysis can split into two different approaches, local and global. Local methods approximate the sensitivity of the response variable to small changes about some nominal point in the random space (D.G.Cacuci, 2003). Global methods compute the same sensitivity but by varying the input parameters through their entire range of variation. One approach for computing the global sensitivities is the ANOVA (ANalysis Of VAriance) or Sobol expansion (Sobol, 2001; Sobol', 2003). For a response function $\mathrm{X}(t, \boldsymbol{\xi})$ with $\boldsymbol{\xi} \in[-1,1]^{M}$, the Sobol expansion decomposes the response, at some time $\mathrm{t}$, into summands of increasing dimension, namely: 


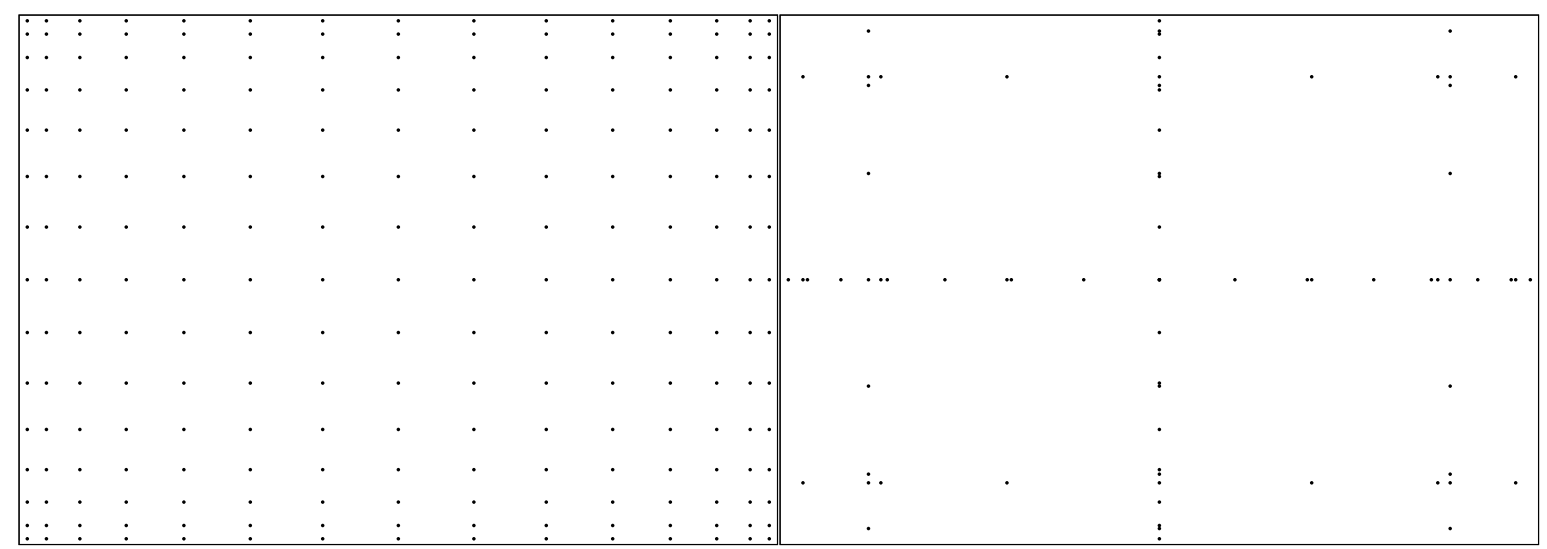

(a) Tensor Product

(b) Sparse Grid

Figure 6: Comparison of tensor product and sparse quadrature grids for a $\mathcal{L}=3$ Gauss-Legendre rule.

$$
\begin{aligned}
\mathrm{X}(t, \boldsymbol{\xi}) & =\mathrm{X}_{0}+\sum_{i=1}^{M} \mathrm{X}_{i}\left(t, \xi_{i}\right) \\
& +\sum_{1 \leq i_{1}<i_{2} \leq M} \mathrm{X}_{i_{1}, i_{2}}\left(t, \xi_{i_{1}}, \xi_{i_{2}}\right) \\
& +\cdots+\mathrm{X}_{i_{1}, \cdots, i_{M}}\left(t, \xi_{i_{1}}, \cdots, \xi_{i_{M}}\right),
\end{aligned}
$$

which can also be written in a more compact form:

$$
\mathrm{X}(t, \boldsymbol{\xi})=\sum_{\boldsymbol{u} \subseteq \mathcal{D}} \mathrm{x}_{\boldsymbol{u}}\left(t, \boldsymbol{\xi}_{\boldsymbol{u}}\right)
$$

where $\boldsymbol{u} \subseteq \mathcal{D}$, where $\mathcal{D}:=\{1, \cdots, M\}$ denotes the set of coordinate indices and $\mathrm{x}_{\emptyset}\left(\boldsymbol{\xi}_{\emptyset}\right)=\mathrm{x}_{0}$. The cardinality of the set $\boldsymbol{u}$ is defined as $|\boldsymbol{u}|$ and $\boldsymbol{\xi}_{\boldsymbol{u}}$ denotes the $|\boldsymbol{u}|$ dimensional vector containing those components of $\boldsymbol{\xi}$ that belong to the set $\boldsymbol{u}$, i.e. $\boldsymbol{\xi}_{\boldsymbol{u}}=$ $\left(\xi_{i}\right)_{i \in \boldsymbol{u}}$. The variance, or total variance, can also be written in terms of a hierarchical expansion in the form of Equation (59), namely

$$
\begin{aligned}
v_{T}(t) & =\operatorname{Var}[\mathrm{X}(\boldsymbol{\xi}, t)]=\int_{[-1,1]^{M}} \mathrm{X}^{2}(\boldsymbol{\xi}, t) p(\boldsymbol{\xi}) d \boldsymbol{\xi}-\mathrm{X}_{0}^{2}(t) \\
& =\sum_{\boldsymbol{u} \subseteq \mathcal{D}, \boldsymbol{u} \notin \emptyset} v_{\boldsymbol{u}}(t)
\end{aligned}
$$

where the variance components in Equation (60) can be calculated from the following expression: 


$$
v_{\boldsymbol{u}}=\int_{[-1,1]^{|\boldsymbol{u}|}} \mathrm{X}_{\boldsymbol{u}}^{2}\left(t, \boldsymbol{\xi}_{\boldsymbol{u}}\right) p\left(\boldsymbol{\xi}_{\boldsymbol{u}}\right) d \boldsymbol{\xi}_{\boldsymbol{u}}
$$

The Sobol sensitivity indices are defined as:

$$
S_{\boldsymbol{u}}=\frac{v_{\boldsymbol{u}}}{v_{T}}
$$

and by definition:

$$
\sum_{\boldsymbol{u} \subseteq \mathcal{D}, \boldsymbol{u} \notin \emptyset} S_{\boldsymbol{u}}=1
$$

Thus, each index $S_{\boldsymbol{u}}$ is a sensitivity measure describing the contribution that the set of variables $\boldsymbol{u}$ have on the total variance. A total sensitivity coefficient $S_{T_{i}}$ can also be defined in order to assess the total effect that a single input parameter, $i$, has on the response and is defined as a sum over all indices that involve parameter $i$, namely

$$
S_{T_{i}}=\sum_{\boldsymbol{u} \subseteq \mathcal{D}, \boldsymbol{u} \notin \emptyset, i \in \boldsymbol{u}} S_{\boldsymbol{u}}
$$

Thus, the total sensitivity coefficient embodies the sole influence of parameter $i$ as well as the cooperative effect with any number of other parameters. It is straight forward to show that Equation (64) may be written as:

$$
S_{T_{i}}=1-\sum_{\boldsymbol{u} \subseteq \mathcal{D}, \boldsymbol{u} \notin \emptyset, i \notin \boldsymbol{u}} S_{\boldsymbol{u}}
$$

where the summation now involves all indexes $\boldsymbol{u}$ that do not contain the index $i$.

The Sobol sensitivities are usually calculated using Monte Carlo (Saltelli et al., 2000) which requires significant computation effort particularly when the number of dimensions, $M$, is large. An alternative method, which is based upon GPC, has been proposed in Sudret (2008) and used to solve time independent structural mechanics problems (Blatman and Sudret, 2010; Crestaux et al., 2009) and also dynamical problems (Sandoval et al., 2012). To calculate the sensitivity indices from the polynomial chaos coefficients we must first re-order the expansion in (40) and collect terms involving an equal number of dimensions. To do this, we define the following index sets

$$
\mathcal{F}_{i_{1}, \cdots, i_{s}}=\left\{\begin{array}{cccc}
\boldsymbol{\alpha}: & \alpha_{k}>0 & \forall k=1, \cdots M & k \in\left(i_{1}, \cdots, i_{s}\right) \\
\alpha_{k}=0 & \forall k=1, \cdots M & k \notin\left(i_{1}, \cdots, i_{s}\right)
\end{array}\right\},
$$


Thus, the set $\mathcal{F}_{i_{1}, \cdots, i_{s}}$ contains only those polynomials that depend on the parameters $\xi_{i_{1}}, \cdots, \xi_{i_{s}}$. We can now re-write the GPC expansion in (40) as follows:

$$
\begin{aligned}
\mathrm{X}(\boldsymbol{\xi}, t) & =\mathrm{x}_{0}+\sum_{i=1}^{M} \sum_{\boldsymbol{\alpha} \in \mathcal{F}_{i}} \mathrm{x}_{\boldsymbol{\alpha}}(t) \Phi_{\boldsymbol{\alpha}}\left(\xi_{i}\right) \\
& +\sum_{1 \leq i_{1}<i_{2} \leq M} \sum_{\boldsymbol{\alpha} \in \mathcal{F}_{i_{1}, i_{2}}} \mathrm{x}_{\boldsymbol{\alpha}}(t) \Phi_{\boldsymbol{\alpha}}\left(\xi_{i_{1}}, \xi_{i_{2}}\right) \\
& +\cdots+\sum_{\boldsymbol{\alpha} \in \mathcal{F}_{i_{1}, \cdots, i_{M}}} \mathrm{x}_{\boldsymbol{\alpha}}(t) \Phi_{\boldsymbol{\alpha}}\left(\xi_{i_{1}}, \cdots, \xi_{i_{M}}\right)
\end{aligned}
$$

which can be written in the more compact form as

$$
\mathrm{X}(\boldsymbol{\xi}, t)=\sum_{\boldsymbol{u} \subseteq \mathcal{D}} \sum_{\boldsymbol{\alpha} \in \mathcal{F}_{\boldsymbol{u}}} \mathrm{x}_{\boldsymbol{\alpha}}(t) \Phi_{\boldsymbol{\alpha}}\left(\boldsymbol{\xi}_{\boldsymbol{u}}\right)
$$

Equation (68) is the GPC analogue of the Sobol expansion in Equation (59) where each of the components $\mathrm{X}_{\boldsymbol{u}}(t, \boldsymbol{\xi})$ has been expanded using the set polynomial basis functions $\mathcal{F}_{\boldsymbol{u}}$ that solely involve the variable $\boldsymbol{u}$.

The components of the total variance can be calculated using Equation (60) as follows:

$$
\begin{aligned}
v_{\boldsymbol{u}} & =\int_{[-1,1]|\boldsymbol{u}|} \mathrm{X}_{\boldsymbol{u}}^{2}\left(t, \boldsymbol{\xi}_{\boldsymbol{u}}\right) d \boldsymbol{\xi}_{\boldsymbol{u}} \\
& =\sum_{\boldsymbol{\alpha} \in \mathcal{F}_{\boldsymbol{u}}} \mathrm{x}_{\boldsymbol{\alpha}}^{2}(t) \int_{[-1,1]^{|\boldsymbol{u}|}} \Phi_{\boldsymbol{\alpha}}^{2}\left(\boldsymbol{\xi}_{\boldsymbol{u}}\right) d \boldsymbol{\xi}_{\boldsymbol{u}} \\
& =\sum_{\boldsymbol{\alpha} \in \mathcal{F}_{\boldsymbol{u}}} \mathrm{x}_{\boldsymbol{\alpha}}^{2}(t) N_{\boldsymbol{\alpha}}^{2}
\end{aligned}
$$

Therefore the total sensitivity measure $S_{T_{i}}$ in Equation (65) can be written as

$$
S_{T_{i}}=1-\frac{1}{v_{T}} \sum_{\substack{\boldsymbol{u} \subseteq \mathcal{D} \\ \boldsymbol{u} \notin \emptyset \\ i \notin \boldsymbol{u}}} \sum_{\boldsymbol{\alpha} \in \mathcal{F}_{\boldsymbol{u}}} \mathrm{x}_{\boldsymbol{\alpha}}^{2}(t) N_{\boldsymbol{\alpha}}^{2}
$$

\subsection{Results}

In this section we assign an uncertainty to combinations of the parameters $\chi_{i}, \tau$ and $S_{0}$ and observe the resulting uncertainties on the outputs. Unless stated otherwise, all uncertain parameters will have a uniform probability distributions with a standard deviation of $10 \%$ of the reference value quoted in Section 2.2 (meaning there is a uniform probability function between about $83 \%$ and $117 \%$ of the base value) whilst all other parameters will be treated as deterministic and having the value quoted in 
Section 2.2. $\chi_{1}$ will always be deterministic because it is the mean number of neutrons produced per fission and allowing this to be uncertain would allow the value of $k_{\text {eff }}$ to be uncertain, which is the not the goal of this uncertainty analysis.

By default, the simulation will be run until 10s for the cases $k_{\text {eff }}=1.01$ and $k_{\text {eff }}=1.3$ but only to $1 \times 10^{-5} \mathrm{~s}$ for the case $k_{\text {eff }}=0.99$. This is because, for the subcritical case, both the mean and standard deviation of the single chain survival probability tends towards zero and this makes calculating these values and the Relative Standard Deviation (RSD) difficult at late times. In the superctritical cases the mean and standard deviation tend toward to a steady value and so are not affected by this limitation.

\subsubsection{Convergence for the Quadratic Case}

In the quadratic case we set $\chi_{\max }=2$ and assign uncertainty to $\chi_{2}$ only. We run the code for various polynomial chaos orders and three different values of $k_{\text {eff }}$ using a maximum sparse grid collocation scheme level of 6 , which requires the evaluation of 127 points in stochastic space. This is compared against the analytic solution produced by Equations (38) and (39) in Figure 7. This shows that both the polynomial chaos model simulation and the Monte Carlo simulation produce results very similar to the analytic solution. For each $k_{\text {eff }}$ the mean values are qualitatively similar to the corresponding deterministic case in Figure 1 whilst the RSD tends towards approximately $10 \%$.

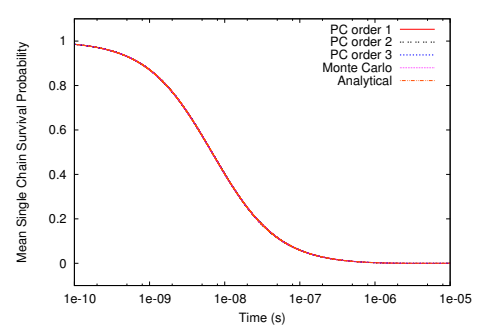

(a) Mean $k_{\text {eff }}=0.99$

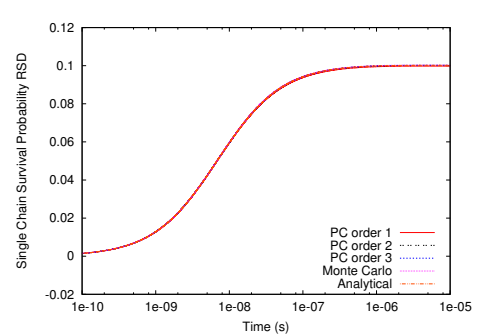

(d) RSD $k_{\text {eff }}=0.99$

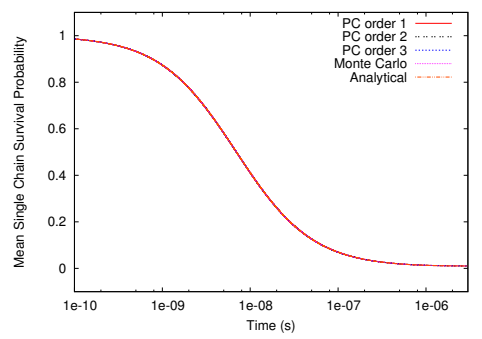

(b) Mean $k_{\text {eff }}=1.01$

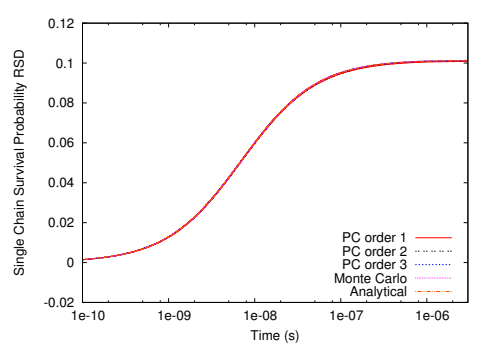

(e) RSD $k_{\text {eff }}=1.01$

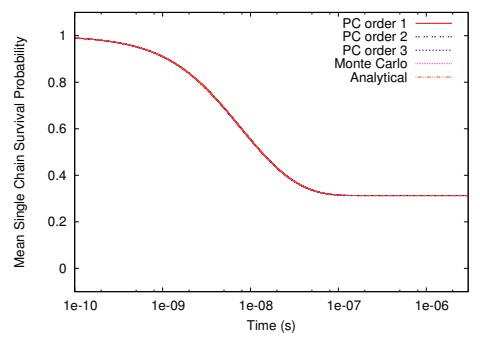

(c) Mean $k_{\text {eff }}=1.3$

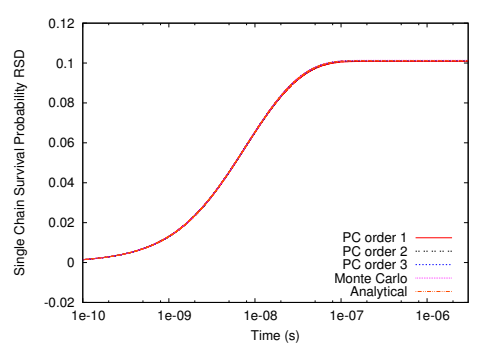

(f) RSD $k_{\text {eff }}=1.3$

Figure 7: The mean and RSD of the probability of survival of a single neutron chain for three different values of $k_{\text {eff }}$ produced (with $\chi_{\max }=2$ and uncertainty assigned to $\chi_{2}$ only) by the polynomial chaos and Monte Carlo and the analytical result.

We may also compute the analytical PDF of the probability of a single neutron chain surviving from the Polynomial Chaos Expansion (PCE). This is achieved by inserting a set of random variables directly into the PCE producing a realisation of the response. Repeating this procedure many times yields an ensemble of realisations which may be "binned" to form a PDF. 
We compare this analytic PDF to that produced by the polynomial chaos and Monte Carlo simulations at various different times in Figure 8. It can be seen that the polynomial chaos result for order 1 provides a poor approximation whilst order 2 and order 3 provide much better approximations to the analytical result. The Monte Carlo simulation was carried out with 100,000 realisations and retains significant variation compared to the analytical result, as might be expected from randomly sampled data, although it does still approximate the analytic result.

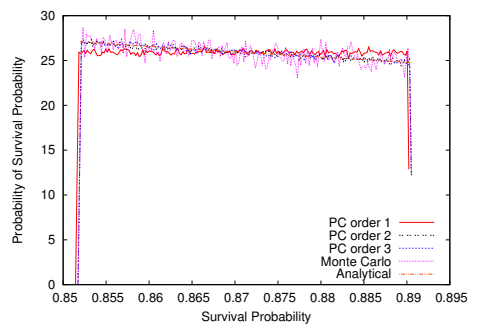

(a) $k_{\text {eff }}=0.99, t=10^{-9} \mathrm{~s}$

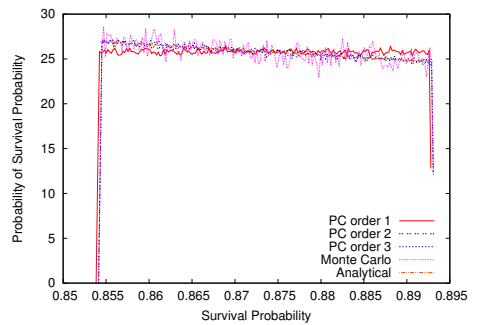

(d) $k_{\text {eff }}=1.01, t=10^{-9} \mathrm{~s}$

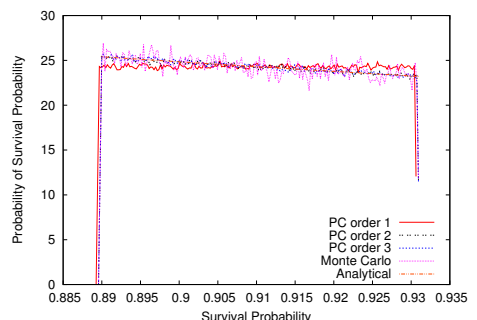

(g) $k_{\text {eff }}=1.3, t=10^{-9} \mathrm{~s}$

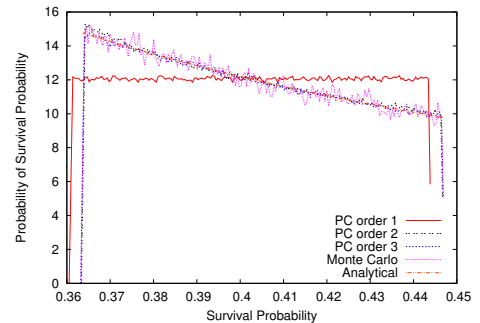

(b) $k_{\text {eff }}=0.99, t=10^{-8} \mathrm{~s}$

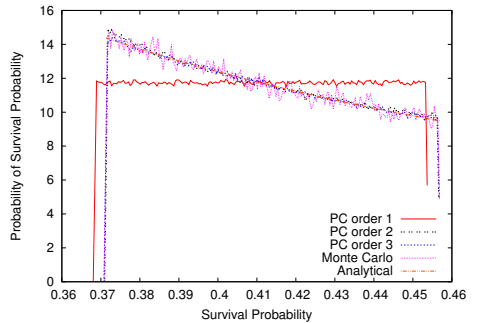

(e) $k_{\text {eff }}=1.01, t=10^{-8} \mathrm{~s}$

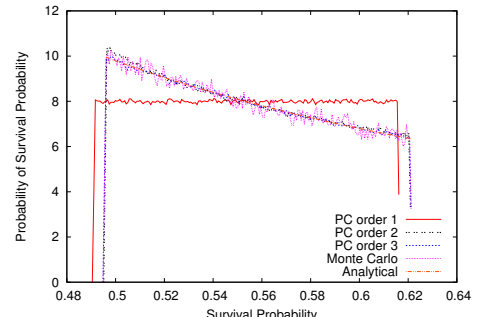

(h) $k_{\text {eff }}=1.3, t=10^{-8} s$

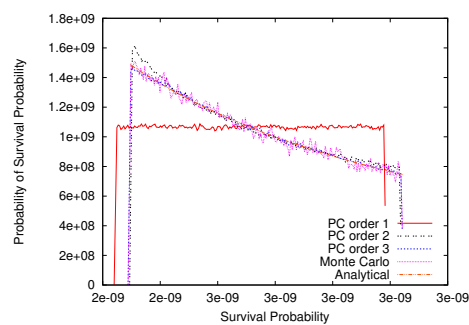

(c) $k_{\text {eff }}=0.99, t=10^{-5} \mathrm{~s}$

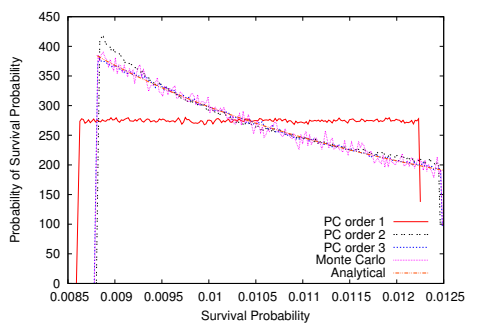

(f) $k_{\text {eff }}=1.01, t=10^{-5} \mathrm{~s}$

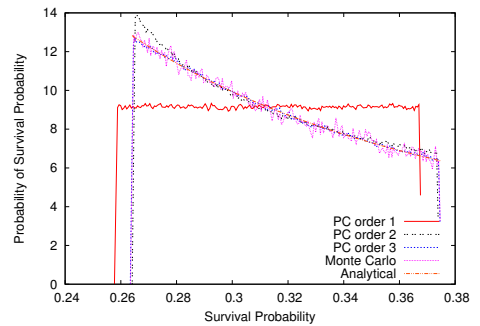

(i) $k_{\text {eff }}=1.3, t=10^{-5} \mathrm{~s}$

Figure 8: The PDF of the probability of one neutron chain surviving until a given time with $\chi_{\max }=2$ and uncertainty assigned to $\chi_{2}$ only. Each method of simulation was used to produce a probability function with 200 bins.

We may examine the convergence and the computational efficiency of the system in this case as there is an analytical solution for both the mean and standard deviation. The measure of the error we choose to use is defined in Equation (71):

$$
\epsilon=\frac{\int_{\ln \left(t_{\text {min }}\right)}^{\ln \left(t_{\max }\right)} \frac{\left(\left|X_{s}(t)\right|_{t=\exp u}-\left|X_{a}(t)\right|_{t=\exp u} \mid\right)}{\left.X_{a}(t)\right|_{t=\exp u}} \mathrm{~d} u}{\ln \left(t_{\text {min }}\right)-\ln \left(t_{\text {max }}\right)},
$$

where $\epsilon$ is the error measure, $u$ is the logarithm of time, $X_{s}(t)$ is the simulated result, $X_{a}(t)$ is the analytical result, $t_{\min }$ is the smallest time at which simulated data is output 
from the model $\left(1 \times 10^{-10} \mathrm{~s}\right)$ and $t_{\max }$ is the time at which the simulation ends $(10 \mathrm{~s}$ for the supercritical cases and $1 \times 10^{-5} \mathrm{~s}$ for the subcritical case). $X$ may represent the mean or standard deviation of the single chain survival probability. This formulation ensures that the contribution to the error between $1 \times 10^{-10} \mathrm{~s}$ and $1 \times 10^{-9} \mathrm{~s}$ is given the same weight as the contribution to the error made between $1 \times 10^{-5} \mathrm{~s}$ and $1 \times 10^{-6} \mathrm{~s}$. This is important as the change in variables happens over a range of timescales. The error as a function of number of realisations is shown in Figure 9 and as a function of computational running time in Figure 10.

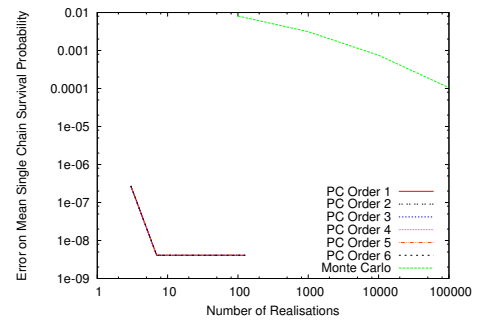

(a) $k_{\text {eff }}=0.99$

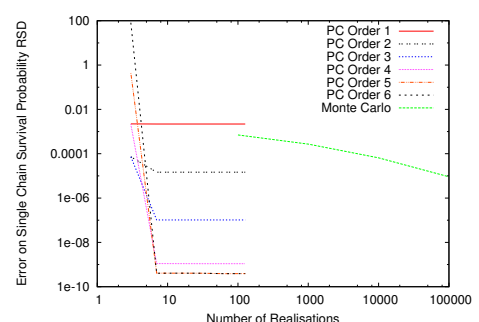

(d) $k_{\text {eff }}=0.99$

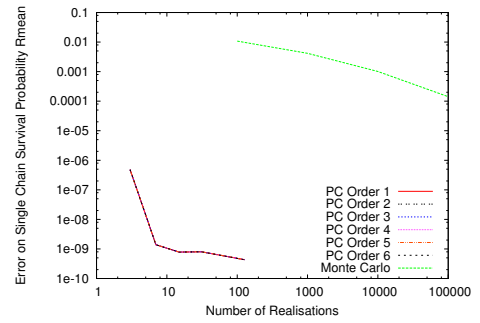

(b) $k_{\text {eff }}=1.01$

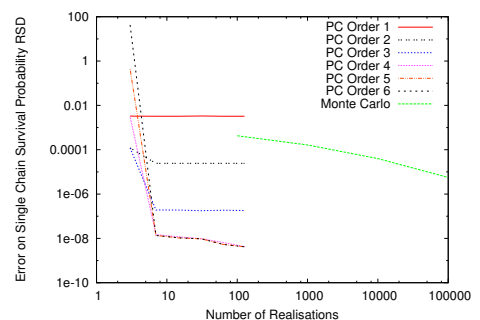

(e) $k_{\text {eff }}=1.01$

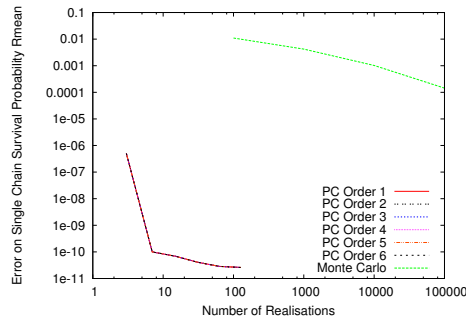

(c) $k_{\text {eff }}=1.3$

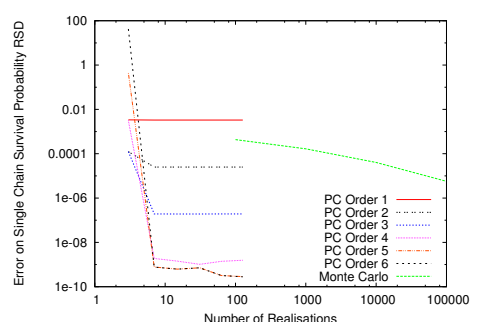

(f) $k_{\text {eff }}=1.3$

Figure 9: The error as defined by Equation (71) for different values of $k_{\text {eff }}$ for both the mean and standard deviation of the single neutron chain survival probability as a function of the number of realisations. For the polynomial chaos case different numbers of realisations are achieved by altering the level of the collocation scheme.

Figures 9 and 10 show that, providing there are at least 7 realisations (i.e. the use of collocation scheme level 2 or above), the polynomial chaos scheme does not converge significantly with higher numbers of realisations. When the collocation scheme is used at level 1 (3 realisations) the evaluations of the mean and standard deviation are very poor, particularly for higher PC orders. This is to eb expected because, for each polynomial order a number of points equal to the polynomial order plus one is required to fit a curve precisely and so, for polynomial order 3 and higher collocation scheme 2 or higher is required to a system with sufficient constraints to fit a meaningful set of polynomials. Further points than are required may still improve the fit (as is observed) by providing more information when evaluating the coefficients of the polynomial.

Increasing PC order was observed to generally decrease the error in standard deviation more than the mean as the mean is already fairly well converged at $\mathrm{PC}$ order 1. Increasing the polynomial order past 4 provides small or no gains in accuracy to the standard deviation. This may be because of the limitations in machine accuracy preventing further convergence being observed. However, by this stage the mean and 


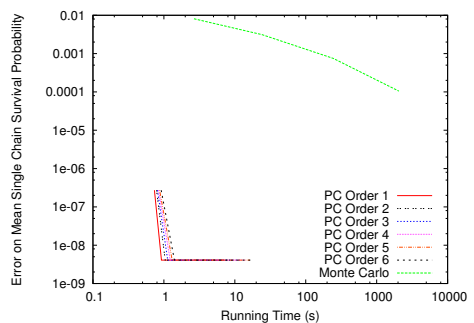

(a) $k_{\text {eff }}=0.99$

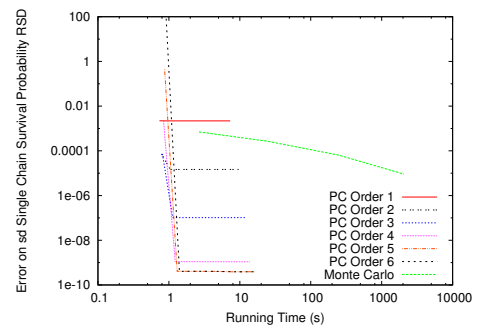

(d) $k_{\text {eff }}=0.99$

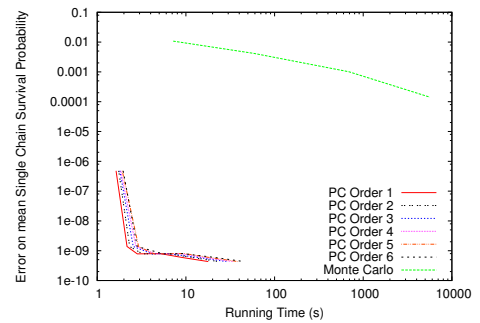

(b) $k_{\text {eff }}=1.01$

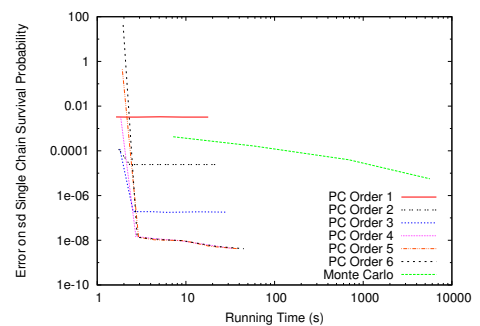

(e) $k_{\text {eff }}=1.01$

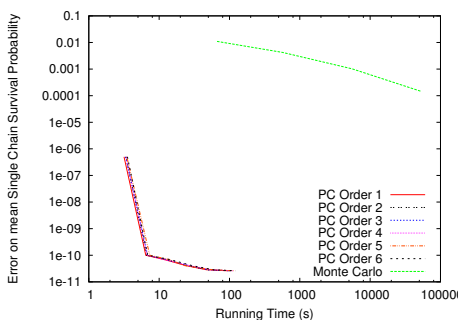

(c) $k_{\text {eff }}=1.3$

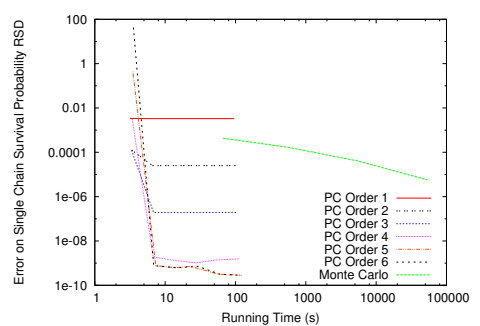

(f) $k_{\text {eff }}=1.3$

Figure 10: The error as defined by Equation (71) for different values of $k_{\text {eff }}$ for both the mean and standard deviation of the single neutron chain survival probability as a function of computational running time.

standard deviation are sufficiently converged for all practical purposes.

For a given collocation scheme the increase in computational time with increasing $\mathrm{PC}$ order is small compared to the increase in computational time which accompanies an increase in collocation scheme level. This implies the majority of computational time is, in this case, associated with evaluating each realisation instead of with the overheads associated with projecting on the polynomials and calculating statistics.

The Monte Carlo simulations sees a slow improvement in accuracy as the number of realisations is increased. However, it is clear that they are not as accurate or efficient as the polynomial chaos simulations (providing a high enough level collocation scheme is used) and would require a very large number of realisations at a high computational cost before am accuracy comparable to that of the polynomial chaos simulations could be achieved.

\subsubsection{Uncertainty on all Parameters}

We now allow all parameters ( $\chi_{i}$ for $2 \leq i \leq 7, \tau$ and $S_{0}$ ) be simultaneously uncertain. To keep running times reasonable with the increased number of stochastic dimensions, the polynomial chaos order of these simulations will be 3 and the collocation scheme level used will be 5 (equivalent to 54,673 points). It was found that a high level collocation scheme was needed to provide a sufficiently large number of points to reduce the error associated with integration over the stochastic dimensions for the purposes of calculating sensitivities to different input parameters. The means and RSDs for both the single chain survival probability and the source extinction probability for the three values of $k_{\text {eff }}$ are shown in Figure 11, alongside the corresponding results for 100,000 Monte Carlo realisations. 


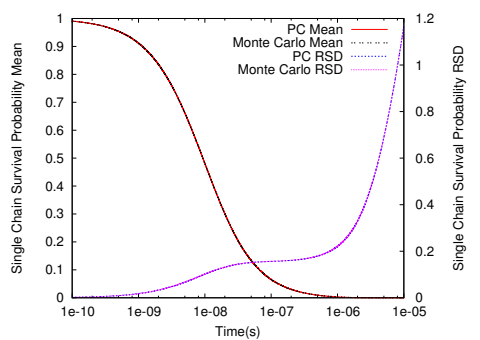

(a) $k_{\text {eff }}=0.99$

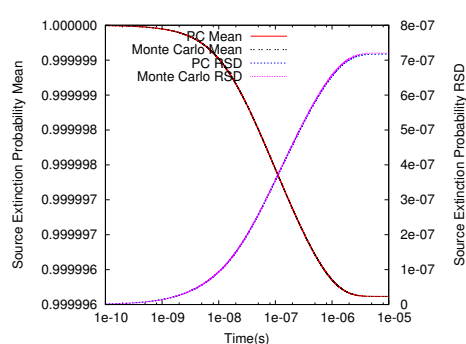

(d) $k_{\text {eff }}=0.99$

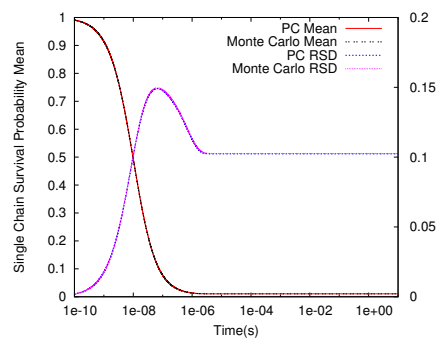

(b) $k_{\text {eff }}=1.01$

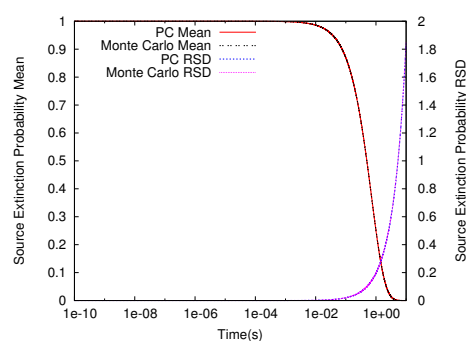

(e) $k_{\text {eff }}=1.01$

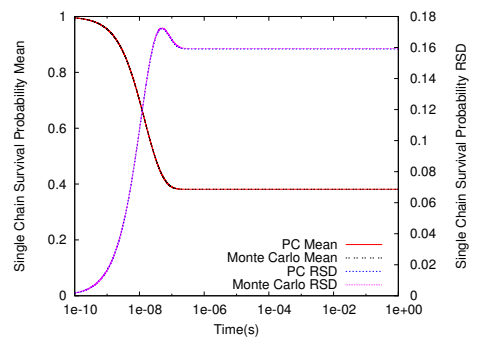

(c) $k_{\text {eff }}=1.3$

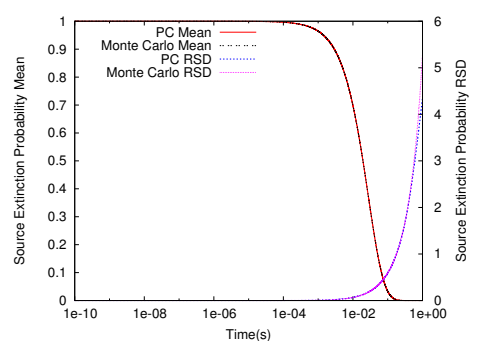

(f) $k_{\text {eff }}=1.3$

Figure 11: The means and RSDs of the single chain survival probability and source extinction probability for the three values of $k_{\text {eff }}$ studied with all parameters uncertain.

Figure 11 shows that the means appear very similar to the deterministic results in Figure 2, indicating that the qualitative response is not drastically changed by the added uncertainty. The RSDs all tend towards a steady value if the mean tends towards a steady value (the single chain survival probability for the super-critical cases and the source extinction probability in the sub-critical case). In the other cases the RSD begins to rise as time continues, becoming very large. This occurs because differences in how quickly the value in question tends towards zero can cause the ratio of the maximum and minimum values of the response surface as a function of $\tau$ to grow which, in turn, increases the RSD. This also tends to amplify variation between the polynomial chaos results and the Monte Carlo simulation caused by a finite polynomial chaos order or number of Monte Carlo realisations. Indeed, significant deviation between the two sets of results is only found in cases where the mean tends towards zero. Otherwise, the agreement between polynomial chaos and Monte Carlo is good.

We may also examine the PDF for the survival of a single neutron chain in this cases where all parameters are uncertain. These results are shown in Figure 12. In each case the Monte Carlo and Polynomial Chaos simulations are in good agreement. In each case the PDF is approximately centred on the relevant mean value at that time found in Figure 11. The PDFs bear some resemblance to those in Figure 8 although, in this case, they tend to be more rounded due to uncertainty in a larger number of parameters.

The Sobol sensitivity of the results of the simulations to each of the uncertain parameters may also be studied, as described in Section 5.2. These results for each of the values of $k_{\text {eff }}$ studied are shown in Figure 13.

The first observation is, as might be expected, the sensitivity to $\chi_{i}$ increases as $i$ 


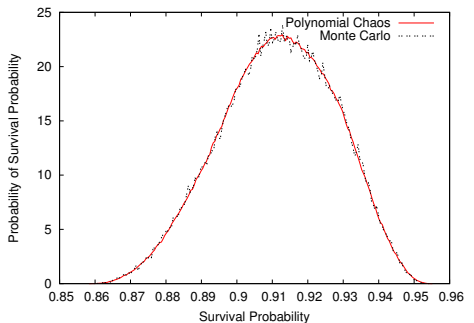

(a) $k_{\text {eff }}=0.99, t=10^{-9} s$

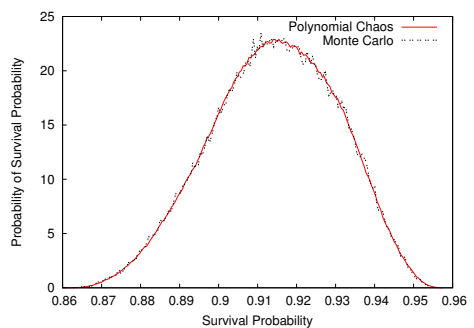

(d) $k_{\text {eff }}=1.01, t=10^{-9} \mathrm{~s}$

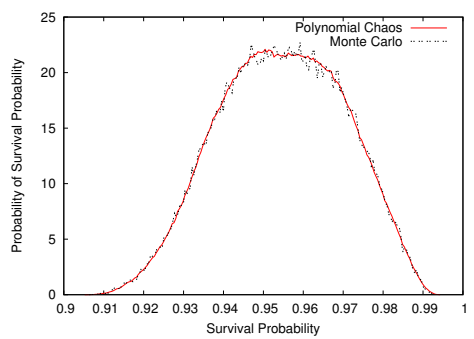

(g) $k_{\text {eff }}=1.3, t=10^{-9} \mathrm{~s}$

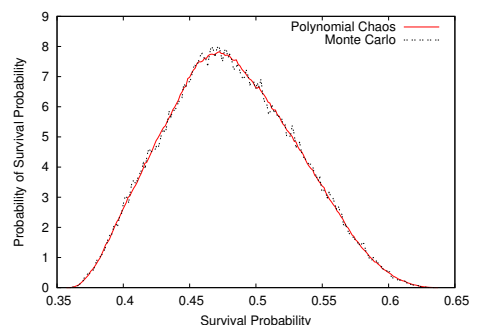

(b) $k_{\text {eff }}=0.99, t=10^{-8} \mathrm{~s}$

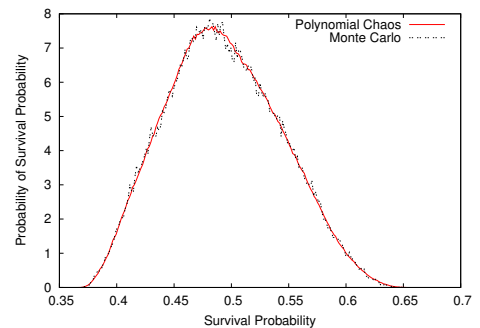

(e) $k_{\text {eff }}=1.01, t=10^{-8} \mathrm{~s}$

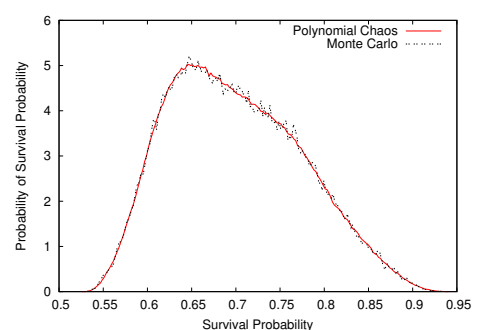

(h) $k_{\text {eff }}=1.3, t=10^{-8} \mathrm{~s}$

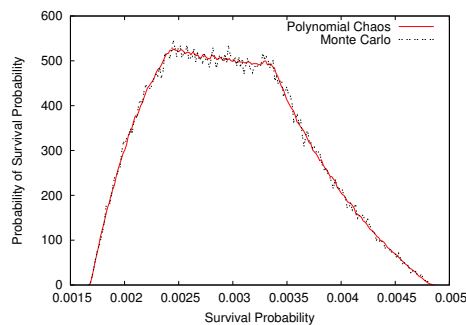

(c) $k_{\text {eff }}=0.99, t=10^{-6} s$

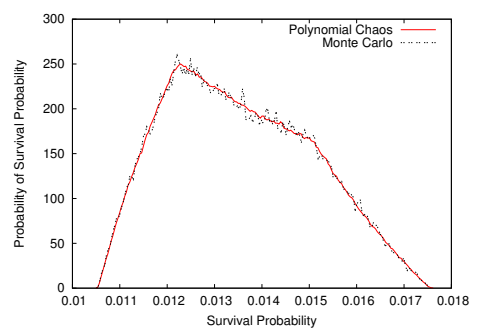

(f) $k_{\text {eff }}=1.01, t=10^{-6} \mathrm{~s}$

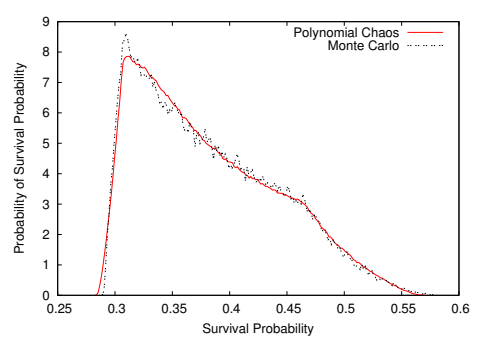

(i) $k_{\text {eff }}=1.3, t=10^{-6} \mathrm{~s}$

Figure 12: The PDF for the survival probability of a single chain of neutrons for the three values of $k_{\text {eff }}$ studied with all parameters uncertain. All Monte Carlo simulations performed with 100,000 realisations.

decreases. Mathematically this is because the contribution due to $\chi_{i}$ in Equation (1) is proportional to $\frac{P(t)^{i}}{i !}$ which decreases with increasing $i$. Physically this is because $\chi_{i}$ with large $i$ is related to the unlikely cases where a fission produces a large number of neutrons (see Table 1 and 3).

In the subcritical case the single chain survival probability is most sensitive to $\chi_{2}$ at low $t$. This is because $\chi_{2}$ is the primary characterisation of the neutron multiplicity distribution and this distribution dictates the probability of a neutron chain surviving a given number of generations. At higher $t$ the probability of a chain surviving is very low and falling, with the rate at which it is falling controlled primarily by the neutron lifetime.

For the subcritical case the source extinction probability is governed by the average time a neutron chain will survive and the rate at which new neutron chains are instigated. The former is governed by $\chi_{2}$ and $\tau$ whilst the latter is governed by $S_{0}$ and these three parameters have approximately equal sensitivities when $t$ is large. At small $t$ the dependence on $\tau$ is weak because the probability of a neutron chain having 


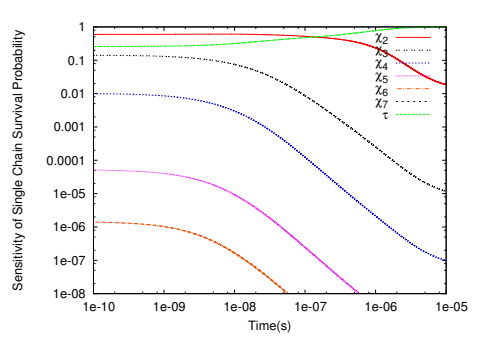

(a) $k_{\text {eff }}=0.99$

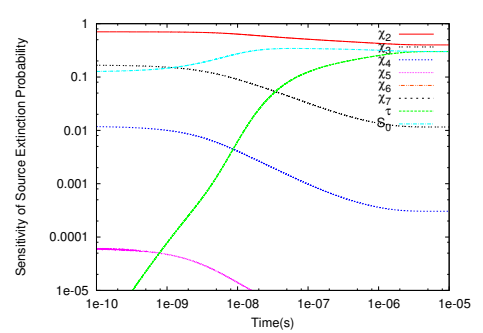

(d) $k_{\text {eff }}=0.99$

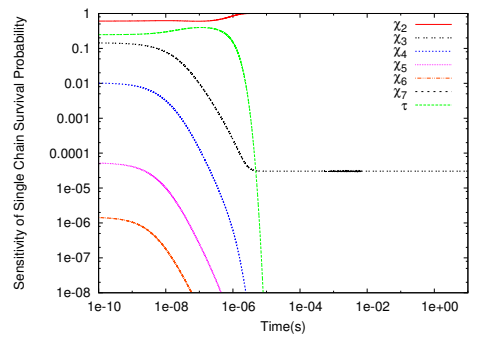

(b) $k_{\text {eff }}=1.01$

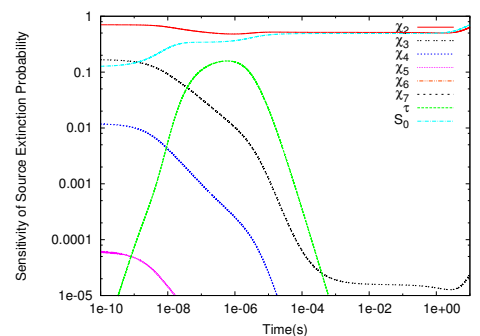

(e) $k_{\text {eff }}=1.01$

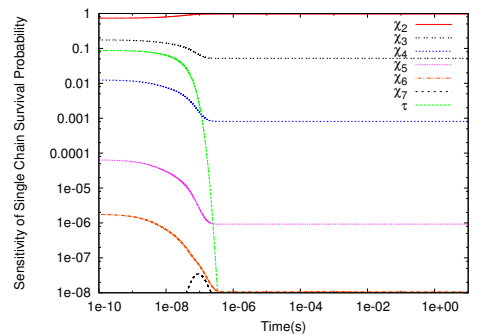

(c) $k_{\text {eff }}=1.3$

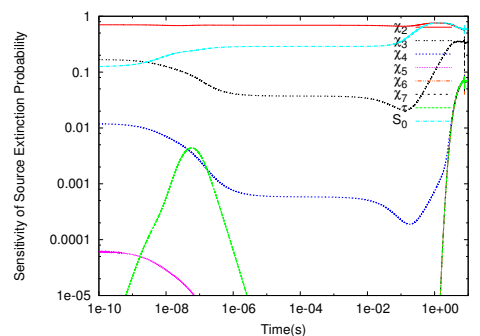

(f) $k_{\text {eff }}=1.3$

Figure 13: The sensitivity of the single chain survival and source extinction probabilities for each of the values of $k_{\text {eff }}$ studied

been initiated is initially zero and rises as time progresses.

In the supercritical cases we see that the single chain survival probability is most sensitive to $\chi_{2}$ at all values of $t$. This is because $\chi_{2}$ is the primary characterisation of the neutron multiplicity distribution and this distribution dictates the probability of a neutron chain surviving a given number of generations. However, at low times, the single chain survival probability is also sensitive to the neutron lifetime $\tau$ which determines how many generations have occurred in a given time period. As time becomes larger, however, the number of neutron generations which have passed becomes less important as a given neutron chain will have either died out or multiplied into such a large number of neutrons it will not die out. As a result, the corresponding sensitivity to $\tau$ falls.

The supercritical source extinction probability is most sensitive to the values of $\chi_{2}$ and $S_{0}$. At the time when the single neutron chain survival probability is decreasing most quickly $\left(\sim 1 \times 10^{-7} \mathrm{~s}\right)$ the neutron lifetime is also important. As time progresses however, the source extinction probability tends to zero. At this point the neutron lifetime becomes irrelevant and the sensitivity to it decreases rapidly. For the $k_{\text {eff }}=1.3$ case the sensitivity to all parameters except $\chi_{2}$ and $S_{0}$ begins to rise at around $t=1 \mathrm{~s}$. There's no obvious physical reason for this and, given that the sensitivity to a large number of parameters rises to the same value at the same rate at this time it seems likely that this is caused by numerical issues which occur as the source extinction probability becomes very small. 


\section{Conclusions}

The deterministic method of solving the equations has been proven accurate when compared to both Monte Carlo and analytical results. These results showed, as might be expected, that a single neutron chain in a subcritical system will always be absorbed whilst, in a supercritical system, the survival probability will tend to a finite value. In the subcritical case the source extinction probability will tend towards a finite value dependent upon the rate at which neutrons are injected and how long a neutron chain lasts on average before being absorbed. In the supercritical case the source extinction probability case always tends towards zero as eventually one of the injected neutrons will result in a chain which multiplies to a large number of neutrons.

Once the method of GPC is used to model the effects of uncertainty the methods are, again, found to agree well with both the Monte Carlo and analytic solutions. It is found that, whilst a relatively low number of Monte Carlo realisations provides a result which is quantitatively similar to the analytic solution in terms of mean and RSD, a much larger number of realisations are required to produce a qualitatively similar probability distribution or a well converged mean and RSD in a quantitative sense. Meanwhile, the method of GPC provides a well converged mean for very low polynomial orders and collocation scheme levels. The RSD, however, becomes significantly more accurate as the polynomial order increases, but not as the collocation scheme level increases (providing sufficient realisations are evaluated to perform the integration in stochastic space). Overall, the GPC method is significantly more computationally efficient that the Monte Carlo simulation.

It is found that a high order collocation scheme level is require to provide enough points to accurately evaluate the sensitivities when all parameters are uncertain. It is found that the neutron lifetime is important in the subcritical case and in the supercritical case where the time is of the same order as the neutron lifetime. The source strength is important in all cases for the neutron source extinction probability. The values of $\chi_{i}$ are important for low values of $i$ in almost all cases. When several parameters are important simultaneously, it is common for the simulation to have a similar sensitivity to each important parameter.

Future work in this area would involve the migration from a mono-energetic, spaceindependent model to a more complex model. This would allow the study of the effects of uncertainty of other parameters including nuclear cross-sections as well as an analysis of the effect of the location of the source which initialises the neutron chains.

\section{Acknowledgements}

Mr Dan Ayres would like to acknowledge the support of EPSRC and Imperial College London through the Knowledge Transfer Secondment scheme (KTS). Dr Matthew Eaton would like to acknowledge the support of the Royal Academy of Engineering through their fellowship programme. The authors would also like to acknowledge EPSRC through their support of the EPSRC grant Adaptive hierarchical radiation transport methods to meet future challenges in reactor physics (EPSRC grant no. EP/J002011/1). 
G. Bell. Probability distribution of neutrons and precursors in a multiplying assembly. Annals of Physics, 21(5):243-283, 1963.

G. Bell. On the stochastic theory of neutron transport. Nuclear Science and Engineering, 21(5):390-401, 1965.

G. Blatman and B. Sudret. Efficient computation of global sensitivity indices using sparse polynomial chaos expansions. Reliability Engineering Eamp; System Safety, 95(11):1216-1229, November 2010.

R. Cameron and W. Martin. The orthogonal development of nonlinear functionals in series of fourier-hermite functionals. Annals of Mathematics, 48(2):385-392, 1947.

T. Crestaux, O. Le Maitre, and J. Martinez. Polynomial chaos expansion for sensitivity analysis. Reliability Engineering 65 System Safety, 94:1161-1172, 2009.

D.G.Cacuci. Sensitivity and Uncertainty Analysis. Chapman and Hall, 2003.

B.C. Diven, H.C. Martin, R.F. Taschek, and J. Terrell. Multiplicities of fission neutrons. Physical Review, 101(3):1012-1015, 1956.

T. Gerstner and M. Griebel. Numerical integration using sparse grids. Numerical Algorithms, 18:209-232, 1998.

R. Ghanem. Ingredients for a general purpose stochastic finite elements implementation. Computer Methods in Applied Mechanics and Engineering, 168:19-34, 1999.

F. Nobile, R. Tempone, and C. G. Webster. A sparse grid stochastic collocation method for partial differential equations with random input data. SIAM Journal on Numerical Analysis, 46:2309-2345, 2008.

J.D. Orndoffl. Prompt neutron periods of metal critical assemblies. Nuclear Science and Engineering, 2(3):450-460, 1957.

S. Park, M.M.R. Williams, A.K. Prinja, and M.D. Eaton. Modelling non-Gaussian uncertainties and the KarhunenLoéve expansion within the context of polynomial chaos. Annals of Nuclear Energy, 76:146-165, 2015.

I. Pazsit and L. Pal. Neutron Fluctuations: A Treatise on the Physics of Branching Processes. Elsevier, Oxford, 2008.

A. K. Prinja and F. J. Souto. Probability distributions for neutron multiplying systems without delayed neutrons. Technical report, Los Alamos, 2009. LA-UR-09-07886.

A. K. Prinja and F. J. Souto. Some considerations on stochastic neutron populations. Transactions of the American Nuclear Society, 102(5):255-257, 2010.

Anil K. Prinja. Notes on the lumped backward master equation for the neutron extinction/survival probability. Technical report, Los Alamos, 2012. LA-UR-12-22638. 
A. Radkowsky. Naval reactor physics handbook: Volume 1. Technical report, United States Atomic Energy Commission, 1964.

A. Saltelli, K. Chan, and E. M. Scott. Sensitivity Analysis. Wiley, 2000.

E. Sandoval, F. Anstett-Collin, and M. Basset. Sensitivity study of dynamic systems using polynomial chaos. Reliability Engineering \& System Safety, 104:15 - 26, 2012.

L. Shampine and M. Gordon. Computer Solutions of Ordinary Differential Equations: The Initial Value Problem. Chapman \& Hall, New York, 1975.

S.A. Smolyak. Quadrature and interpolation formulas for tensor products of certain classes of functions. Doklady Akademii Nauk SSSR, 4:240-243, 1963.

I.M Sobol. Global sensitivity indices for nonlinear mathematical models and their monte carlo estimates. Mathematics and Computers in Simulation, 55:271 - 280, 2001.

I.M. Sobol'. Theorems and examples on high dimensional model representation. Reliability Engineering 85 System Safety, 79:187 - 193, 2003.

P. Spanos and R. Ghanem. Stochastic finite element expansion for random media. Journal of Engineering Mechanics, 115(5):1035-1053, 1989.

G. Stefanou. The stochastic finite element method: Past, present and future. Computer Methods in Applied Mechanics and Engineering, 198(9-12):1031-1051, 2009.

B. Sudret. Global sensitivity analysis using polynomial chaos expansions. Reliability Engineering Eamp; System Safety, 93(7):964-979, July 2008.

G. W. Wasilkowski and H. Wozniakowski. Explicit cost bounds of algorithms for multivariate tensor product problems. Journal of Complexity, 11:1-56, 1995.

N. Wiener. The homogeneous chaos. American Journal of Mathematics, 60:897-936, 1938.

M.M.R. Williams. Random Processes in Nuclear Reactors. Pergamon Press, Oxford, 1974.

D. Xiu and J. Hesthaven. High-order collocation methods for differential equations with random inputs. SIAM Journal on Scientific Computing, 27:1118-1139, 2005.

D. Xiu and G.E. Karniadakis. The wiener-askey polynomial chaos for stochastic differential equations. SIAM Journal on Scientific Computing, 24(2):619-644, 2002.

M.S. Zucker and N.E. Holden. Energy dependence of the neutron multiplicity pv in fast neutron induced fission of 235,238u and 239pu. Technical report, Brookhaven National Laboratory, 1986. 